\title{
A Finite Element Level Set Redistancing Method Based on Gradient Recovery
}

\author{
Arnold Reusken*
}

Bericht Nr. 346

Oktober 2012

Key words: level set method, reinitialization, redistancing, gradient recovery

AMS Subject Classifications: 65M06, 65D05

Institut für Geometrie und Praktische Mathematik RWTH Aachen

Templergraben 55, D-52056 Aachen (Germany)

* Institut für Geometrie und Praktische Mathematik, RWTH Aachen University, D-52056 Aachen, Germany; email: reusken@igpm.rwth-aachen.de 


\title{
A FINITE ELEMENT LEVEL SET REDISTANCING METHOD BASED ON GRADIENT RECOVERY
}

\author{
ARNOLD REUSKEN*
}

\begin{abstract}
We introduce a new redistancing method for level set functions. This method applies in a finite element setting and uses a gradient recovery technique. Based on the recovered gradient a quasi-normal field on the zero level of the finite element level set function is defined and from this an approximate signed distance function is determined. For this redistancing method rigorous error bounds are derived. For example, the distance between the original zero level and the zero level after redistancing can be shown to be bounded by $c h^{k+1}$, if finite elements of degree $k$ are used in the discretization.
\end{abstract}

Key words. level set method, reinitialization, redistancing, gradient recovery

AMS subject classifications. 65M06, 65D05

1. Introduction. The level set method is a very popular method to treat problems involving free surfaces or interfaces. We refer to the literature for an explanation of this method and of fields of application, e.g. [16, 17, 12, 18]. Very often in this level set method a so-called reinitialization or redistancing (or reparametrization) procedure is used. Since the introduction of the reinitialization approach in [4] many of such methods have been proposed in the literature. Most of these can be classified as either PDE-based $[19,14]$ or geometry based $[15,17,5]$. We refer to the recent paper [3] for a discussion and comparison of these approaches. For an approach based on extension velocities, in which reinitialization is avoided, we refer to $[1,6]$.

In reinitialization methods, for a given discrete approximation $\phi_{h}$ of the unknown level set function $\phi$ one constructs an approximate signed distance function $d_{h}$ to the (only implicitly given) zero level $\Gamma_{h}$ of $\phi_{h}$. Let the (implicitly given) zero level of $d_{h}$ be denoted by $\tilde{\Gamma}_{h}$. In general, for an approximate signed distance function one has $\operatorname{dist}\left(\tilde{\Gamma}_{h}, \Gamma_{h}\right)>0$. In reinitialization methods one tries to construct a function $d_{h}$ such that, in a neighborhood of $\Gamma_{h}$, this function is "close to" the exact signed distance function to $\Gamma_{h}$, denoted by $d_{h}^{e x}$, and such that $\operatorname{dist}\left(\tilde{\Gamma}_{h}, \Gamma_{h}\right)$ is "small". In numerical analyses of such methods one typically uses numerical experiments, for certain model problems, to investigate and quantify what is meant by "close to" and "small". We are not aware of any paper in which rigorous error bounds for the difference between $d_{h}$ and $d_{h}^{e x}$ or for $\operatorname{dist}\left(\tilde{\Gamma}_{h}, \Gamma_{h}\right)>0$ are derived.

In this paper we present a new, geometry based, reinitialization method and derive rigorous error bounds for it. Although its error analysis is rather technical, the basic idea of the method is easy to explain. Our method is a geometric one, with a structure that is common in fast marching techniques (FMM), namely an initial phase combined with an extension phase. In the initial phase one assigns values to the finite element nodes of the simplices that are intersected by $\Gamma_{h}$. These values are close to (as quantified further on) the signed distance values to $\Gamma_{h}$. Given these values, in the extension phase values are assigned to all other nodes, using e.g. a fast marching sweeping approach . It is well-known that in such redistancing methods the initial phase is the critical one and determines the accuracy of the reinitialization method. The method that we introduce is different from the methods known in the

*Institut für Geometrie und Praktische Mathematik, RWTH-Aachen University, D-52056 Aachen, Germany; email: reusken@igpm.rwth-aachen.de 
literature with respect to its initial phase. For the extension phase one can use any of the extension methods already available in the literature.

We explain the approach used in the initial phase. First consider a point $z$ that is close to the zero level $\Gamma$ of a smooth level set function $\phi$. The normals on $\Gamma$ are denoted by $n$. We then have a unique decomposition $z=p(z)+d^{e x}(z) n(p(z))$, with $p(z) \in \Gamma$ the orthogonal projection of $z$ on $\Gamma$. By definition, the distance from $z$ to $\Gamma$ is given by $\left|d^{e x}(z)\right|=\|z-p(z)\|$. We adapt this elementary distance characterization to the discrete case. We assume that $\Gamma_{h}$ is the zero level of a finite element approximation $\phi_{h}$ of $\phi$. The function $\phi_{h}$ is continuous and a piecewise polynomial of degree $k$ (on simplices). Under weak assumptions, $\Gamma_{h}$ is a Lipschitz-manifold. We introduce the notion of a quasi-normal field $n_{h}$ on such a Lipschitz-manifold. Its essential conditions are that $x \rightarrow n_{h}(x), x \in \Gamma_{h}$, is locally Lipschitz-continuous and that $n_{h}(x)$ is "close to" orthogonal to $\Gamma_{h}$ (cf. Definition 3.1 for precise formulation). The "close to orthogonal" condition is quantified using a parameter $\delta$; in the case of a smooth manifold one has $\delta=|\cos \theta|$, with $\theta$ the angle between $n_{h}(x)$ and the tangent plane at $x$. Based on this quasi-normal field, for $z$ sufficiently close to $\Gamma_{h}$ there is a unique representation $z=p(z)+d_{h}(z) n_{h}(p(z)), p(z) \in \Gamma_{h}$, which induces an approximate signed distance function $d_{h}(z)$. In general $z \rightarrow p(z)$ is an oublique projection on $\Gamma_{h}$. In a general analysis, presented in section 4 , the difference between $d_{h}(z)$ and the exact signed distance value $d_{h}^{e x}(z)$ to $\Gamma_{h}$ can be bounded in terms of the orthogonality parameter $\delta$. To be able to apply this abstract approach in a concrete finite setting we need such a quasi-normal field. The normal field $\nabla \phi_{h}(x) /\left\|\nabla \phi_{h}(x)\right\|, x \in \Gamma_{h},(x$ not on a simplex boundary) does not satisfy the requirements, since it is discontinuous between simplices, hence not Lipschitz. In the finite element literature, however, there are so-called gradient recovery techniques which result in approximations of $\nabla \phi_{h}$ that satisfy the conditions of a quasi-normal field on $\Gamma_{h}$. In this paper, as an exampe, we use the polynomial-preserving recovery (PPR) technique introduced in [20]. Using error bounds for this recovery technique and (discretization) error bounds for $\phi_{h}-\phi$ we can quantify statements like " $d_{h}$ is close to $d_{h}^{\text {ex" }}$ and "dist $\left(\tilde{\Gamma}_{h}, \Gamma_{h}\right)$ is small". We prove, for example that $\operatorname{dist}\left(\tilde{\Gamma}_{h}, \Gamma_{h}\right) \leq c h^{k+1}$ and $\left\|I_{h} d_{h}-d_{h}^{e x}\right\|_{L^{\infty}\left(\Omega_{\Gamma_{h}}\right)} \leq c h^{k+1}$ hold, with $I_{h}$ the nodal finite element interpolation operator and $\Omega_{\Gamma_{h}}$ the neigborhood of $\Gamma_{h}$ consisting of all simplices that are intersected by $\Gamma_{h}$. Thus, for quadratic finite elements $(k=2)$ our reinitialization method has accuracy $h^{3}$. We include results of a numerical experiment (in 3D) that illustrates this $h^{3}$ error behavior.

2. Lipschitz-manifolds. In the literature there are several (not all equivalent) definitions of $k$-dimensional Lipschitz-manifolds in $\mathbb{R}^{N}$. A recent overview of the commonly used ones is given in [10]. In this paper we restrict ourselves to the case $k=N-1$ and use the standard definition of an $(N-1)$-dimensional Lipschitz-manifold in graph representation, cf. e.g. $[8,10]$, which we now recall.

Definition 2.1. A subset $\mathcal{M} \subset \mathbb{R}^{N}$ is called an $(N-1)$-dimensional Lipschitzmanifold (in graph representation) if for every $x_{0} \in \mathcal{M}$ there exists a local Euclidean coordinate system with origin $O_{x_{0}}$ in $x_{0}$ and such that

1. there are open subsets $V_{1} \in \mathbb{R}^{N-1}$ and $V_{2} \subset \mathbb{R}$ with $O_{x_{0}} \in V:=V_{1} \times V_{2}$,

2. there exists a Lipschitz mapping $h: V_{1} \rightarrow V_{2}$ with

$$
\mathcal{M} \cap V=\left\{\left(x_{1}, h\left(x_{1}\right)\right) \mid x_{1} \in V_{1}\right\} .
$$

In view of our application to the level set function we also introduce the following im- 
plicit representation of an $(N-1)$-dimensional Lipschitz-manifold which is equivalent to the one given in Defition 2.1, cf. Theorem 2.11 in [10].

Definition 2.2. A subset $\mathcal{M} \subset \mathbb{R}^{N}$ is called an $(N-1)$-dimensional Lipschitzmanifold (in implicit representation) if for every $x_{0} \in \mathcal{M}$ there exists a local Euclidean coordinate system with origin $O_{x_{0}}$ in $x_{0}$ and such that

1. there are open subsets $V_{1} \in \mathbb{R}^{N-1}$ and $V_{2} \subset \mathbb{R}$ with $O_{x_{0}} \in V:=V_{1} \times V_{2}$,

2. there exists a scalar function $\phi: V \rightarrow \mathbb{R}$ with $\phi\left(O_{x_{0}}\right)=0$ and there are constants $L_{\phi}, K_{\phi}>0$ such that

$$
\begin{aligned}
|\phi(x)-\phi(y)| & \leq L_{\phi}|| x-y \| \quad \text { for all } x, y \in V, \\
\left|\phi\left(x_{1}, x_{2}\right)-\phi\left(x_{1}, y_{2}\right)\right| & \geq K_{\phi}\left|x_{2}-y_{2}\right| \quad \text { for all } x_{1} \in V_{1}, x_{2}, y_{2} \in V_{2},
\end{aligned}
$$

3. $\mathcal{M} \cap V=\{x \in V \mid \phi(x)=0\}$.

In the remainder we assume $\Gamma$ to be compact, and thus an open cover of $\Gamma$ resulting from the definitions above can be reduced to a finite cover.

3. A Lipschitz continuous local coordinate system. If $\Gamma$ is a $C^{2}$-manifold then the (oriented) normal field $n(x), x \in \Gamma$, can be used to define a local coordinate system of the form

$$
y=p(y)+d(y) n(p(y)), \quad y \in\left\{y \in \mathbb{R}^{N}|| d(y) \mid<\epsilon\right\},
$$

with $\epsilon>0$ sufficiently small, $d(\cdot)$ the signed distance function to $\Gamma$ and $p(\cdot)$ the orthogonal projection onto $\Gamma$. In this section we derive a generalization of this local coordinate system for the case of a Lipschitz-manifold. We introduce a so-called quasinormal field and a corresponding approximate signed distance function. These form the basis for the reinitialization method discussed in section 6 .

We use $B(x ; r) \subset \mathbb{R}^{N}$ to denote the open ball with centre $x \in \mathbb{R}^{N}$ and radius $r>0$ and $B_{\Gamma}(x ; r):=B(x ; r) \cap \Gamma$.

In the remainder, $\Gamma$ is assumed to be an $(N-1)$-dimensional Lipschitz-manifold.

DEFINITION 3.1. A quasi-normal field is a mapping $n: \Gamma \rightarrow \mathbb{R}^{N}$, with $\|n(x)\|=1$ for all $x$, which has the following properties. For all $x \in \Gamma$ there exist $\delta_{x}<1, r_{x}>0$ and $\gamma_{x}, c_{x}$ with:

$$
\begin{aligned}
& \|n(x)-n(y)\| \leq \gamma_{x}\|x-y\| \quad \text { for all } y \in B_{\Gamma}\left(x ; r_{x}\right), \\
& |\langle n(x), x-y\rangle| \leq \delta_{x}\|x-y\|+c_{x}\|x-y\|^{2} \quad \text { for all } y \in B_{\Gamma}\left(x ; r_{x}\right), \\
& \text { and } \sup _{x \in \Gamma} \delta_{x}=: \delta<1, \quad \sup _{x \in \Gamma} \gamma_{x}<\infty, \quad \sup _{x \in \Gamma} c_{x}<\infty, \quad \inf _{x \in \Gamma} r_{x}>0 .
\end{aligned}
$$

The conditions in (3.2) and (3.3) can be interpreted as smoothness and transversality conditions that the field $n$ has to satisfy. For a $C^{2}$-manifold these conditions are satisfied for the normal field (with $\delta_{x}=\mathcal{O}\left(r_{x}\right)$ ). For a quasi-normal field we can always reduce $r_{x}$ (if necessary) such that

$$
0<r_{x} \leq \frac{1-\delta_{x}}{4\left(\gamma_{x}+c_{x}\right)}
$$

holds. With $r_{x}$ such that this holds and $y \in B_{\Gamma}\left(x ; r_{x}\right)$ property (3.3) implies that $|\langle n(x), x-y\rangle| \leq \frac{1}{2}(1+\delta)\|x-y\|$ and thus the angle between $n(x)$ and $x-y$ is bounded from below by a strictly positive constant, uniformly in $x$ and $y$. Given a quasi-normal field $n$ we define:

$$
F: \Gamma \times \mathbb{R} \rightarrow \mathbb{R}^{N}, \quad F(x, t):=x+\operatorname{tn}(x) .
$$


We first derive the local injectivity of this function:

Lemma 3.1. Given a quasi-normal field with $r_{x}$ such that (3.5) is satisfied, define

$$
D:=\left\{(x, t) \mid x \in \Gamma, t \in\left(-\frac{1}{2} r_{x}, \frac{1}{2} r_{x}\right)\right\} .
$$

The function $F$ is injective on $D$.

Proof. Take $(x, t),(\hat{x}, \hat{t}) \in D$ such that $F(x, t)=F(\hat{x}, \hat{t})$, i.e. $x+\operatorname{tn}(x)=\hat{x}+$ $\hat{t} n(\hat{x})$. If $x=\hat{x}$ it follows that $t=\hat{t}$. Assume that $x \neq \hat{x}$. Note that $\|F(x, t)-\hat{x}\|=|\hat{t}|$ and $\|F(\hat{x}, \hat{t})-x\|=|t|$. Define $m:=\max \left\{r_{x}, r_{\hat{x}}\right\}$. Note that

$$
\|x-\hat{x}\|=\|\hat{t} n(\hat{x})-t n(x)\| \leq|\hat{t}|+|t|<\frac{1}{2}\left(r_{\hat{x}}+r_{x}\right) \leq m
$$

holds. It sufficies to consider the case $m=r_{x}$. Thus $\hat{x} \in B_{\Gamma}\left(x, r_{x}\right)$ holds. From (3.3) and (3.2) we obtain

$$
\begin{aligned}
|\langle n(x), x-\hat{x}\rangle| & \leq\left(\delta_{x}+c_{x} r_{x}\right)\|x-\hat{x}\| \leq \frac{1}{2}\left(1+\delta_{x}\right)\|x-\hat{x}\| \\
|\langle n(\hat{x}), x-\hat{x}\rangle| & \leq|\langle n(\hat{x})-n(x), x-\hat{x}\rangle|+|\langle n(x), x-\hat{x}\rangle| \\
& \leq\left(\gamma_{x} r_{x}+\delta_{x}+c_{x} r_{x}\right)\|x-\hat{x}\| \leq \frac{1}{2}\left(1+\delta_{x}\right)\|x-\hat{x}\| .
\end{aligned}
$$

From $t=\hat{t}=0$ we obtain the contradiction $x=\hat{x}$. Hence $|t|+|\hat{t}|>0$ holds. From this and the results in (3.8)-(3.9) it follows that the triangle with vertices $x$, $\hat{x}$ and $F(x, t)=F(\hat{x}, \hat{t})$ is non-degenerated. The inner angle at vertex $x$ is denoted by $\beta$. From (3.8) we obtain $|\cos \beta| \leq \frac{1}{2}\left(1+\delta_{x}\right)$ and thus $\sin \beta>1-|\cos \beta| \geq$ $\frac{1}{2}\left(1-\delta_{x}\right)$ holds. The inner angle at $F(x, t)=F(\hat{x}, \hat{t})$ is denoted by $\alpha$. The cosine rule implies $\|n(x)-n(\hat{x})\|^{2}=2(1-\cos \alpha)=4 \sin ^{2}\left(\frac{1}{2} \alpha\right)$ and thus $\sin \alpha \leq 2 \sin \left(\frac{1}{2} \alpha\right)=$ $\|n(x)-n(\hat{x})\| \leq \gamma_{x}\|x-\hat{x}\|$ holds. Due to the sine rule we have $\frac{|\hat{t}|}{\sin \beta}=\frac{\|x-\hat{x}\|}{\sin \alpha}$, and thus $|\hat{t}|=\frac{\sin \beta}{\sin \alpha}\|x-\hat{x}\|>\frac{1}{2}\left(1-\delta_{x}\right) \gamma_{x}^{-1}$ holds. This yields a contradiction with $|\hat{t}|<\frac{1}{2} r_{x} \leq \frac{1}{2}\left(1-\delta_{x}\right) \gamma_{x}^{-1}$, cf. (3.7). Hence $x=\hat{x}$, which implies $t=\hat{t}$, and thus $(x, t)=(\hat{x}, \hat{t})$ holds.

Take $z \in F(D)$, with $D$ as in Lemma 3.1. There are unique $p(z)=x \in \Gamma, d(z) \in$ $\left(-\frac{1}{2} r_{x}, \frac{1}{2} r_{x}\right)$ such that

$$
z=p(z)+d(z) n(p(z)) .
$$

Theorem 3.2. Take $D$ as in lemma 3.1. Then $F(D) \subset \mathbb{R}^{N}$ is open. The functions $z \rightarrow p(z)$ and $z \rightarrow d(z)$ are continuous on $F(D)$.

Proof. Take $F\left(x_{0}, t_{0}\right)=x_{0}+t_{0} n\left(x_{0}\right) \in F(D)$, i.e., $x_{0} \in \Gamma,-\frac{1}{2} r_{x_{0}}<t_{0}<\frac{1}{2} r_{x_{0}}$. From the graph representation of $\Gamma$, cf. Definition 2.1, it follows that there are open subsets $V_{1} \subset \mathbb{R}^{N-1}, V_{2} \subset \mathbb{R}$ and a Lipschitz function $h$ such that $\Gamma \cap V=$ $\left\{\left(x_{1}, h\left(x_{1}\right)\right) \mid x_{1} \in V_{1}\right\}$ with $V=V_{1} \times V_{2}$. Define $r_{V_{1}}:=\inf \left\{r_{x} \mid x=\left(x_{1}, h\left(x_{1}\right)\right), x_{1} \in\right.$ $\left.V_{1}\right\}>0$ and the open subset $U:=V_{1} \times\left(-\frac{1}{2} r_{V_{1}}, \frac{1}{2} r_{V_{1}}\right) \subset \mathbb{R}^{N}$. On $U$ we define the continuous mapping $G\left(x_{1}, t\right):=F\left(\left(x_{1}, h\left(x_{1}\right)\right), t\right) \in F(D)$. Lemma 3.1 implies injectivity of the function $G$. From Brouwer's invariant domain theorem it follows that $G(U) \subset F(D)$ is open and the inverse of $G^{-1}: G(U) \rightarrow U$ is continuous. Hence, $F(D)$ is open. The inverse is given by $G^{-1}(z)=\left(x_{1}, t\right)$ with $x_{1}$ and $t$ such that $z=\left(x_{1}, h\left(x_{1}\right)\right)+t n\left(x_{1}, h\left(x_{1}\right)\right)$. Continuity of $G^{-1}$ implies that the mapping $z \rightarrow t=d(z)$ is continuous. Furthermore, using the continuity of $h$ we also obtain the 
continuity of $z \rightarrow\left(x_{1}, h\left(x_{1}\right)\right)=p(z)$.

The compact Lipschitz-manifold $\Gamma$ is contained in the open set $F(D)$ and thus for $z$ sufficiently close to $\Gamma$ we have a unique decomposition $z=p(z)+d(z) n(p(z))$. Note that the width of the domain $D$ of the mapping $F$ is determined by $r_{x}$, which in turn depends on the parameters $\delta_{x}, \gamma_{x}, c_{x}$ that quantify the local smoothness and transversality of the quasi-normal field $n$ and the local geometry ("curvature") of the manifold $\Gamma$.

Based on the representation in (3.10) there is the following natural definition of an approximate distance function.

DEFINITION 3.2. The function $z \rightarrow d(z)$, with $d$ as in (3.10) is called the approximate signed distance function.

Note that this function $d(z)$ gives an approximation of the signed distance, only for $z$ close to the manifold $\Gamma$. Below it will be convenient to use the relation

$$
d(z)=\langle z-p(z), n(p(z))\rangle,
$$

which immediately follows form (3.10). The decomposition in (3.10) corresponds to a local Lipschitz continuous coordinate system and is a generalization of the one for a smooth manifold given in (3.1). Note that $z \rightarrow p(z)$ is an oublique projection onto $\Gamma$.

4. Properties of the approximate signed distance function. In this section we derive some properties of the approximate signed distance function $d(z)$, $z \in F(D)$, defined in (3.10). For convenience we extend the quasi-normal field $n$, by taking a constant value in the quasi-normal direction, as follows:

$$
n(z)=n(p(z)+d(z) n(p(z)):=n(p(z)) \text { for all } z \in D .
$$

The normal field and the manifold $\Gamma$ are characterized by Lipschitz functions. From a Lipschitz version of the implicit function theorem one can conclude that the functions $p$ and $d$ are not only continuous (cf. Theorem 3.2) but even locally Lipschitz continuous. Instead of applying such a theorem we derive this property directly, using the continuity result from Theorem 3.2 and the properties in (3.2)-(3.3). This derivation yields bounds which show how the local Lipschitz constant depends on the orthogonality parameter $\delta$ in (3.3).

Lemma 4.1. For every $z \in F(D)$ there exists an $\epsilon>0$ such that for all $z_{1}, z_{2} \in$ $B(z ; \epsilon)$ the following holds:

$$
\begin{aligned}
\left\|p\left(z_{1}\right)-p\left(z_{2}\right)\right\| & \leq \frac{4}{1-\delta_{p\left(z_{1}\right)}}\left\|z_{1}-z_{2}\right\| \\
\left|d\left(z_{1}\right)-d\left(z_{2}\right)\right| & \leq \frac{2\left(1+\delta_{p\left(z_{1}\right)}\right)}{1-\delta_{p\left(z_{1}\right)}}\left\|z_{1}-z_{2}\right\| .
\end{aligned}
$$

Proof. Take $z_{0}=F\left(x_{0}, t_{0}\right)=x_{0}+t_{0} n\left(x_{0}\right)$ with $x_{0} \in \Gamma, t_{0} \in\left(-\frac{1}{2} r_{x_{0}}, \frac{1}{2} r_{x_{0}}\right)$. Note that $x_{0}=p\left(z_{0}\right)$ and $t_{0}=d\left(z_{0}\right)$. Without loss of generality we can assume that for $x \in \Gamma$ the function $x \rightarrow r_{x}$, with $r_{x}$ as in Definition 3.1, and such that (3.5) holds, is continuous. Due to this and the continuity of the functions $p$ and $d$ there exists an $\epsilon>0$ such that for all $z \in B\left(z_{0} ; \epsilon\right)$ we have $p(z) \in B_{\Gamma}\left(x_{0} ; \frac{1}{4} r_{x_{0}}\right), d(z) \in\left(-\frac{1}{2} r_{x_{0}}, \frac{1}{2} r_{x_{0}}\right)$ and $\max \left\{r_{p(z)} \mid z \in B\left(z_{0} ; \epsilon\right)\right\} \leq 2 \min \left\{r_{p(z)} \mid z \in B\left(z_{0} ; \epsilon\right)\right\}$. Take arbitrary $z_{1}, z_{2} \in$ $B\left(z_{0} ; \epsilon\right)$. From

$$
\left\|p\left(z_{1}\right)-p\left(z_{2}\right)\right\| \leq\left\|p\left(z_{1}\right)-x_{0}\right\|+\left\|p\left(z_{2}\right)-x_{0}\right\|<\frac{1}{2} r_{x_{0}} \leq r_{p\left(z_{1}\right)}
$$


it follows that $p\left(z_{2}\right) \in B_{\Gamma}\left(p\left(z_{1}\right) ; r_{p\left(z_{1}\right)}\right)$ holds. Thus, using (3.2) and (3.3) we get

$$
\begin{aligned}
\left|d\left(z_{1}\right)-d\left(z_{2}\right)\right| & =\left|\left\langle z_{1}-p\left(z_{1}\right), n\left(p\left(z_{1}\right)\right)\right\rangle+\left\langle z_{2}-p\left(z_{2}\right), n\left(p\left(z_{2}\right)\right)\right\rangle\right| \\
& \leq\left|\left\langle p\left(z_{2}\right)-p\left(z_{1}\right), n\left(p\left(z_{1}\right)\right)\right\rangle\right|+\left|\left\langle p\left(z_{2}\right)-z_{2}, n\left(p\left(z_{2}\right)\right)-n\left(p\left(z_{1}\right)\right)\right\rangle\right| \\
& +\left|\left\langle z_{1}-z_{2}, n\left(p\left(z_{1}\right)\right)\right\rangle\right| \\
& \leq\left[\delta_{p\left(z_{1}\right)}+c_{p\left(z_{1}\right)} r_{p\left(z_{1}\right)}+\left|d\left(z_{2}\right)\right| \gamma_{p\left(z_{1}\right)}\right]\left\|p\left(z_{1}\right)-p\left(z_{2}\right)\right\|+\left\|z_{1}-z_{2}\right\| .
\end{aligned}
$$

Note that $\left|d\left(z_{2}\right)\right| \leq \frac{1}{2} r_{x_{0}} \leq r_{p\left(z_{1}\right)}$, and using (3.5) we get

$$
\begin{aligned}
\left|d\left(z_{1}\right)-d\left(z_{2}\right)\right| & \leq\left[\delta_{p\left(z_{1}\right)}+\left(c_{p\left(z_{1}\right)}+\gamma_{p\left(z_{1}\right)}\right) r_{p\left(z_{1}\right)}\right]\left\|p\left(z_{1}\right)-p\left(z_{2}\right)\right\|+\left\|z_{1}-z_{2}\right\| \\
& \leq \frac{1}{4}\left(1+3 \delta_{p\left(z_{1}\right)}\right)\left\|p\left(z_{1}\right)-p\left(z_{2}\right)\right\|+\left\|z_{1}-z_{2}\right\| .
\end{aligned}
$$

Using this we obtain

$$
\begin{aligned}
\left\|p\left(z_{1}\right)-p\left(z_{2}\right)\right\| & =\left\|z_{1}-d\left(z_{1}\right) n\left(p\left(z_{1}\right)\right)-z_{2}+d\left(z_{2}\right) n\left(p\left(z_{2}\right)\right)\right\| \\
& \leq\left\|z_{1}-z_{2}\right\|+\left\|\left(d\left(z_{1}\right)-d\left(z_{2}\right)\right) n\left(p\left(z_{1}\right)\right)\right\|+\left\|d\left(z_{2}\right)\left[n\left(p\left(z_{1}\right)\right)-n\left(p\left(z_{2}\right)\right)\right]\right\| \\
& \leq\left\|z_{1}-z_{2}\right\|+\left|d\left(z_{1}\right)-d\left(z_{2}\right)\right|+\left|d\left(z_{2}\right)\right| \gamma_{p\left(z_{1}\right)}\left\|p\left(z_{1}\right)-p\left(z_{2}\right)\right\| \\
& \leq 2\left\|z_{1}-z_{2}\right\|+\left[\frac{1}{4}\left(1+3 \delta_{p\left(z_{1}\right)}\right)+\frac{1}{4}\left(1-\delta_{p\left(z_{1}\right)}\right)\right]\left\|p\left(z_{1}\right)-p\left(z_{2}\right)\right\| \\
& =2\left\|z_{1}-z_{2}\right\|+\frac{1}{2}\left(1+\delta_{p\left(z_{1}\right)}\right)\left\|p\left(z_{1}\right)-p\left(z_{2}\right)\right\| .
\end{aligned}
$$

Since $\delta_{p\left(z_{1}\right)}<1$ we obtain

$$
\left\|p\left(z_{1}\right)-p\left(z_{2}\right)\right\| \leq \frac{4}{1-\delta_{p\left(z_{1}\right)}}\left\|z_{1}-z_{2}\right\|
$$

and using this in (4.3) results in

$$
\left|d\left(z_{1}\right)-d\left(z_{2}\right)\right| \leq \frac{2+2 \delta_{p\left(z_{1}\right)}}{1-\delta_{p\left(z_{1}\right)}}\left\|z_{1}-z_{2}\right\|,
$$

which completes the proof.

From Rademacher's theorem it follows that $p$ and $d$ are Frechet-differentiable almost everywhere on $F(D)$. Let $N_{s} \subset F(D)$ be the subset with $\left|N_{s}\right|=0$ such that $d$ is differentiable at $F(D) \backslash N_{s}$.

For the exact signed distance function to a Lipschitz-manifold, denoted by $d^{\text {ex }}$, it is known that $\left\|\nabla d^{\operatorname{ex}}(z)\right\|=1$ almost everywhere in a neighborhood of the manifold. From (4.2) it follows that for $z \in F(D) \backslash N_{s}$ we have $\|\nabla d(z)\| \leq 2\left(1+\delta_{p(z)}\right)\left(1-\delta_{p(z)}\right)^{-1}$, i.e. $\|\nabla d(z)\| \lesssim 2$ for $\delta_{p(z)} \ll 1$. In the next theorem we derive $\|\nabla d(z)\| \approx 1$ if $\delta_{p(z)} \ll 1$.

THEOREM 4.2. The following holds, with $\delta_{p(z)}<1$ as in (3.3),

$$
1-\frac{16 \delta_{p(z)}}{7+9 \delta_{p(z)}} \leq\|\nabla d(z)\| \leq 1+\frac{16}{7} \frac{\delta_{p(z)}}{1-\delta_{p(z)}} \quad \text { for all } z \in F(D) \backslash N_{s} .
$$


Proof. The Frechet derivative of a function $G: \mathbb{R}^{N} \rightarrow \mathbb{R}^{N}$ is denoted by $D G$, and its representation at $x \in \mathbb{R}^{N}$ by the Jacobian matrix $D G(x) \in \mathbb{R}^{N \times N}$. From $\|n(z)\|=1$ for all $z \in D$ it follows that

$$
\operatorname{Dn}(z)^{T} n(z)=0 \text { for all } z \in F(D) \backslash N_{s}
$$

holds. From the relation (3.11) we obtain, for $z \in F(D) \backslash N_{s}$,

$$
\begin{aligned}
\nabla d(z) & =D(i d-p)(z)^{T} n(p(z))+D(n \circ p)(z)^{T}(z-p(z)) \\
& =\left(I-D p(z)^{T}\right) n(p(z))+D p(z)^{T} \operatorname{Dn}(p(z))^{T}(z-p(z)) .
\end{aligned}
$$

Using (3.10) and (4.5) we get $\operatorname{Dn}(p(z))^{T}(z-p(z))=d(z) \operatorname{Dn}(p(z))^{T} n(p(z))=0$ and thus

$$
\nabla d(z)=n(p(z))-D p(z)^{T} n(p(z)), \quad z \in F(D) \backslash N_{s} .
$$

Hence,

$$
\|\nabla d(z)-n(p(z))\| \leq\left\|D p(z)^{T} n(p(z))\right\| \quad z \in F(D) \backslash N_{s} .
$$

For $w \in \mathbb{R}^{N}$ and $|\epsilon|$ sufficiently small we obtain, due to (3.3),

$$
|\langle n(p(z)), p(z)-p(z+\epsilon w)\rangle| \leq \delta_{p(z)}\|p(z)-p(z+\epsilon w)\|+c_{p(z)}\|p(z)-p(z+\epsilon w)\|^{2},
$$

and thus, for $z \in F(D) \backslash N_{s}$,

$$
\begin{aligned}
\left\|D p(z)^{T} n(p(z))\right\| & =\max _{\|w\|=1}\left\langle w, D p(z)^{T} n(p(z))\right\rangle \\
& =\max _{\|w\|=1}\langle n(p(z)), D p(z) w\rangle \leq \delta_{p(z)}\|D p(z)\|
\end{aligned}
$$

holds. Relation (3.10) implies

$$
D p(z)=I-d(z) D n(p(z)) D p(z)-n(p(z)) \nabla d(z)^{T},
$$

and thus

$$
[I+d(z) D n(p(z))] D p(z)=I-n(p(z)) \nabla d(z)^{T} .
$$

From (3.2) we obtain $\|D n(p(z))\| \leq \gamma_{p(z)}$ for $z \in F(D) \backslash N_{s}$, and due to $|d(z)| \leq \frac{1}{2} r_{p(z)}$ and the condition (3.5) we get $|d(z)| \mid D n(p(z)) \| \leq \frac{1}{8}\left(1-\delta_{p(z)}\right)$ and thus the matrix $I+d(z) \operatorname{Dn}(p(z))$ is invertible and we have the estimate $\left\|[I+d(z) \operatorname{Dn}(p(z))]^{-1}\right\| \leq$ $8\left(7+\delta_{p(z)}\right)^{-1}$. Combining these results we get

$$
\begin{aligned}
\|\nabla d(z)-n(p(z))\| & \leq\left\|D p(z)^{T} n(p(z))\right\| \leq \delta_{p(z)}\|D p(z)\| \\
& \leq \delta_{p(z)}\left\|[I+d(z) \operatorname{Dn}(p(z))]^{-1}\right\|\left\|I-n(p(z)) \nabla d(z)^{T}\right\| \\
& \leq 8 \delta_{p(z)}\left(7+\delta_{p(z)}\right)^{-1}(1+\|\nabla d(z)\|) .
\end{aligned}
$$

Using $\|n(p(z))\|=1$ and $8 \delta_{p(z)}\left(7+\delta_{p(z)}\right)^{-1}<1$ this implies

$$
1-\frac{16 \delta_{p(z)}}{7+9 \delta_{p(z)}} \leq\|\nabla d(z)\| \leq 1+\frac{16}{7} \frac{\delta_{p(z)}}{1-\delta_{p(z)}}
$$


i.e., the result in (4.4).

Note that the bounds in (4.4) are determined (only) by the quantity $\delta_{p(z)}$ from (3.3). For a "close to orthogonal" quasi-normal field we have $\delta_{p(z)} \ll 1$ and thus $\|\nabla d(z)\| \approx 1$ as quantified in (4.4). In our application below we have $\delta_{p(z)}=\mathcal{O}\left(h^{k}\right) \ll 1$, with $h$ a (local) mesh size parameter and $k$ related to the degree of the finite elements used.

The next result quantifies, in which sense the function $d$ is close to the exact signed distance function $d^{\mathrm{ex}}$.

TheOREm 4.3. Take $z \in F(D), z \notin \Gamma$ and let $x_{0} \in \Gamma$ be such that $\left|d^{\operatorname{ex}}(z)\right|=$ $\min _{x \in \Gamma}\|z-x\|=\left\|z-x_{0}\right\|$. Define $L_{x_{0}, z}:=\left\{x_{0}+t\left(z-x_{0}\right) \mid 0 \leq t \leq 1\right\}$ and assume that $L_{x_{0}, z} \cap N_{s}$ has $1 D$ measure zero. Then

$$
0 \leq \frac{|d(z)|-\left|d^{\mathrm{ex}}(z)\right|}{\left|d^{\mathrm{ex}}(z)\right|} \leq \frac{16}{7} \frac{\delta_{L}}{1-\delta_{L}}
$$

holds, with $\delta_{L}:=\max \left\{\delta_{p(y)} \mid y \in L_{x_{0}, z}\right\}<1$.

Proof. From $|d(z)|=\|z-p(z)\|$, with $p(z) \in \Gamma$, it follows that $|d(z)| \geq\left|d^{\text {ex }}(z)\right|$ and thus the left inequality in (4.7) holds. Using the mean value theorem and $d\left(x_{0}\right)=0$ we get

$$
d(z)=\int_{0}^{1} \nabla d\left(x_{0}+t\left(z-x_{0}\right)\right) \cdot\left(z-x_{0}\right) d t .
$$

Hence, using Theorem 4.2 we obtain

$$
\begin{aligned}
|d(z)| & \leq \max _{y \in L_{x_{0}, z}}\|\nabla d(y)\|\left\|z-x_{0}\right\| \leq \max _{y \in L_{x_{0}, z}}\left(1+\frac{16}{7} \frac{\delta_{p(y)}}{1-\delta_{p(y)}}\right)\left|d^{\mathrm{ex}}(z)\right| \\
& =\left(1+\frac{16}{7} \frac{\delta_{L}}{1-\delta_{L}}\right)\left|d^{\mathrm{ex}}(z)\right|
\end{aligned}
$$

which proves the right inequality in (4.7).

5. A method for computing $p(z)$. In this section we introduce a simple iterative procedure for computing the oublique projection $p(z)$. If for a given $z$ the oublique projection $p(z) \in \Gamma$ is known, the value for $d(z)$ can directly be determined from $d(z)=\langle z-p(z), n(p(z))\rangle$. Let $z$ sufficiently close to $\Gamma(z \in F(D))$ be given. We propose the following scheme:

let $x^{0} \in \Gamma \quad($ close to $p(z))$ be given. For $k \geq 0$ :

$$
x^{k+1}:=z-\alpha n\left(x^{k}\right) \text { with } \alpha \in \mathbb{R} \text { such that } x^{k+1} \in \Gamma \text { and }|\alpha| \text { minimal. }
$$

The following theorem shows that this method is well-defined and converges locally.

THEOREM 5.1. Let $z \in F(D)$ with $|d(z)|<\frac{3}{4} \frac{1-\delta_{p(z)}}{\gamma_{p(z)}}$ be given. For $x^{0}$ suffciently close to $p(z)$ the iteration (5.1) is well-defined and the following holds:

$$
\left\|x^{k+1}-p(z)\right\| \leq \frac{4}{3} \frac{\gamma_{p(z)}}{1-\delta_{p(z)}}|d(z)|\left\|x^{k}-p(z)\right\|, \quad k=0,1 \ldots
$$

Proof. Take $z$ as specified above. In a sufficiently small neighborhood $V=V_{1} \times V_{2}$ of $p(z) \in \Gamma$, as in the Definitions 2.1, 2.2, we can use both the graph representation and level set representation of $\Gamma$ :

$$
\Gamma \cap V=\left\{\left(x_{1}, h\left(x_{1}\right)\right) \mid x_{1} \in V_{1}\right\}=\{x \in V \mid \phi(x)=0\} .
$$


The function $f: V_{1} \times V_{2} \rightarrow \mathbb{R}$ given by $f\left(x_{1}, \alpha\right)=\phi\left(z-\alpha n\left(x_{1}, h\left(x_{1}\right)\right)\right)$ has a zero $\left(x_{1}^{*}, \alpha^{*}\right) \in V_{1} \times V_{2}$, with $x_{1}^{*}$ such that $\left(x_{1}^{*}, h\left(x_{1}^{*}\right)\right)=p(z)$ and $\alpha^{*}=d(z)$. From a Lipschitz version of the implicit function theorem, cf. [7], it follows that there exists a neighborhood $W$ of $x_{1}^{*}$ and a function $\alpha: W \rightarrow V_{1}$ such that $f\left(x_{1}, \alpha\left(x_{1}\right)\right)=0$ for all $x_{1} \in W$ and $\Gamma \cap\left(W \times V_{2}\right)=\left\{f\left(x_{1}, \alpha\left(x_{1}\right)\right)=0 \mid x_{1} \in W\right\}$. This implies that for $x^{0} \in \Gamma$ sufficiently close to $p(z)$ there is a unique $\alpha_{1}=\alpha_{1}\left(x^{0}\right) \in V_{1}$ such that

$$
x^{1}=z-\alpha_{1} n\left(x^{0}\right) \in \Gamma .
$$

We conclude that for $k=0$ the iteration (5.1) is well-defined for all $x^{0} \in \Gamma$ with $\left\|x^{0}-p(z)\right\|<\xi$ and $\xi$ sufficiently small. From a continuity argument it follows that for $\xi>0$ sufficiently small we have $x^{k} \in B_{\Gamma}\left(p(z) ; r_{p(z)}\right)$ for $k=0,1$. We use the notation $e^{k}=x^{k}-p(z)$. From (5.3) and $p(z)=z-d(z) n(p(z))$ it follows that

$$
e^{1}=d(z) n(p(z))-\alpha_{1} n\left(x^{0}\right)=d(z)\left(n(p(z))-n\left(x^{0}\right)\right)+\left(d(z)-\alpha_{1}\right) n\left(x^{0}\right)
$$

holds, and thus

$$
\left\|e^{1}\right\|^{2} \leq|d(z)| \gamma_{p(z)}\left\|e^{0}\right\|\left\|e^{1}\right\|+\left|d(z)-\alpha_{1} \|\left\langle n\left(x^{0}\right), e^{1}\right\rangle\right| .
$$

Note that

$$
\left|d(z)-\alpha_{1}\right|=\left|\|p(z)-z\|-\left\|x^{1}-z\right\|\right| \leq\left\|p(z)-x^{1}\right\|=\left\|e^{1}\right\| .
$$

Hence,

$$
\left\|e^{1}\right\| \leq|d(z)| \gamma_{p(z)}\left\|e^{0}\right\|+\left|\left\langle n\left(x^{0}\right), e^{1}\right\rangle\right|
$$

holds. Using $x^{k} \in B_{\Gamma}\left(p(z) ; r_{p(z)}\right)$ and the conditions (3.2)-(3.3) we get

$$
\begin{aligned}
\left|\left\langle n\left(x^{0}\right), e^{1}\right\rangle\right| & =\left|\left\langle n\left(x^{0}\right), p(z)-x^{1}\right\rangle\right| \\
& \leq\left|\left\langle n\left(x^{0}\right)-n(p(z)), p(z)-x^{1}\right\rangle\right|+\left|\left\langle n(p(z)), p(z)-x^{1}\right\rangle\right| \\
& \leq \gamma_{p(z)} r_{p(z)}\left\|e^{1}\right\|+\left(\delta_{p(z)}+c_{p(z)} r_{p(z)}\right)\left\|e^{1}\right\| .
\end{aligned}
$$

Using this we obtain

$$
\left\|e^{1}\right\| \leq|d(z)| \gamma_{p(z)}\left\|e^{0}\right\|+\left[\delta_{p(z)}+\left(c_{p(z)}+\gamma_{p(z)}\right) r_{p(z)}\right]\left\|e^{1}\right\|
$$

and using (3.5) this yields

$$
\left(1-\frac{1}{4}\left(1+3 \delta_{p(z)}\right)\left\|e^{1}\right\| \leq|d(z)| \gamma_{p(z)}\left\|e^{0}\right\| .\right.
$$

Hence, the result (5.2) holds for $k=0$. From the assumption $|d(z)|<\frac{3}{4} \frac{1-\delta_{p(z)}}{\gamma_{p(z)}}$ it follows that $\left\|e^{1}\right\|<\left\|e^{0}\right\|$ and thus the same argument can be applied for $k \geq 1$.

It remains to determine the value of $\alpha$ such that $x^{k+1}=z-\alpha n\left(x^{k}\right) \in \Gamma$ holds, cf. (5.1). In our applications the manifold $\Gamma$ is represented as the zero level of a level set function $\phi$. In that case the value of $\alpha$ can be determined by a line search algorithm applied to $g_{k}(\alpha)=0$, with $g_{k}(\alpha):=\phi\left(z-\alpha n\left(x^{k}\right)\right)$. If $g_{k}$ is (only) Lipschitz, special algorithms should be used, cf. [7, 13]. 
6. Application to the reinitialization of finite element level set functions. In this section we explain how the approximate signed distance function introduced above, cf. Definition 3.2, can be used in a reinitialization method. For a generel discussion of the role of reinitialization techniques in level set methods we refer to the literature, cf. [11, 15, 16, 17].

We consider a finite element setting and a standard level set description of a smooth manifold (interface) in $\mathbb{R}^{d}(d=2,3)$. Let $\phi: \Omega \rightarrow \mathbb{R}$ be a smooth function and $\Omega \subset \mathbb{R}^{d}, \Omega=\Omega_{1} \cup \Omega_{2}, \Gamma=\bar{\Omega}_{1} \cap \bar{\Omega}_{2}=\{x \in \Omega \mid \phi(x)=0\}, \phi(x)<0$ for $x \in \Omega_{1}$, $\phi(x)>0$ for $x \in \Omega_{2}$.

In applications one typically only has a finite element approximation $\phi_{h}$ of $\phi$ available. Examples of such approximations are mentioned in section 7 below. Let $\left\{\mathcal{T}_{h}\right\}_{h>0}$ be a family of shape regular simplicial triangulations of $\Omega$, and $V_{h}$ the corresponding standard finite element space of continuous piecewise polynomials of degree $k \geq 1$ :

$$
V_{h}=\left\{\psi \in C(\Omega) \mid \psi_{\mid T} \in \mathcal{P}_{k} \text { for all } T \in \mathcal{T}_{h}\right\} .
$$

The function $\phi_{h} \in V_{h}$ is a given (sufficiently accurate) approximation of $\phi$. We define $\Gamma_{h}=\left\{x \in \Omega \mid \phi_{h}(x)=0\right\}$. Note that the zero level set $\Gamma_{h}$ is only implicitly described and except for the case $k=1$ (linear finite elements) it can generally not be represented in an explicit form.

We need some further notation. Let $\mathcal{T}_{\Gamma_{h}}$ be the set of all simplices that are intersected by $\Gamma_{h}$. This set is called local triangulation. The corresponding local domain is denoted by $\Omega_{\Gamma_{h}}=\overline{U_{T \in \mathcal{T}_{h}} T}$. To avoid technical details, we assume the generic situation that the intersection of any simplex $T \in \mathcal{T}_{\Gamma_{h}}$ with $\Gamma_{h}$ divides $T$ into two subsets with nonzero $d$-dimensional measure. For a collection of simplices $A$ let $V(A)$ be the set of all vertices of the simplices contained in $A$ and $N(A)$ the set of all finite element nodes of the simplices contained in $A$. Hence, for $k=1$ we have $V(A)=N(A)$ and $V(A) \subset N(A)$ for $k \geq 2$. For a vertex $v$ the union of all simplices that have $v$ as a vertex is denoted by $\omega(v)$. The local triangulation is enlarged by adding the neigboring simplices, resulting in the set

$$
\mathcal{T}_{\Gamma_{h}}^{e} ;=\cup_{v \in V\left(\mathcal{T}_{\Gamma_{h}}\right)} \omega(v),
$$

which is called the extended local triangulation. The corresponding domain is denoted by $\Omega_{\Gamma_{h}}^{e}$. The finite element space $V_{h}$ restricted to the local triangulation is denoted by $V_{h}\left(\Omega_{\Gamma_{h}}\right):=\left\{\psi_{\mid \Omega_{\Gamma_{h}}} \mid \psi \in V_{h}\right\}$. We define $V_{h}\left(\Omega_{\Gamma_{h}}^{e}\right)$ similarly.

We are interested in a reinitialization of the finite element function $\phi_{h}$. One very often used approach is the Fast Marching (FM) technique $[15,11,5]$ in which an initialization phase and an extension phase are distinguished. In the former, every point from a set of near interface grid points (points that are "close to" the interface) is assigned a value that should be close to the value of the exact signed distance function at that grid point. In the finite element setting introduced above, the near interface grid points are given by $N\left(\mathcal{T}_{\Gamma_{h}}\right)$. Given the initialization values an outward marching algorithm is used to assign values to the other grid points. In general the initialization phase is the most critical one, since the location of the zero level of the reinitialization depends only on the values at the near interface grid points. In this paper we only consider this initialization phase. Given this initialization phase. any of the (many) known variants of the FM extension algorithms can be applied.

The initialization phase that we propose in this paper is based on two key components. For the given $\phi_{h}$ its gradient field $\nabla \phi_{h}$ cannot be used as a quasi-normal field 
on $\Gamma_{h}$ since it is discontinuous (at the faces between simplices). In the finite element literature there are well-established gradient recovery techniques. Such techniques are often used in error estimators, cf. e.g. [2]. A famous example is the gradient recovery method introduced by Zienkiewicz and Zhu [21, 22], known as the Superconvergence Patch Recovery (SPR), which forms the basis of the ZZ error estimator. Basically, the SPR uses a least squares fit to the gradient of the finite element function to recover a continuous gradient. Another method, the so-called Polynomial-Preserving Recovery (PPR) is introduced in [20] and analyzed in [9]. In PPR one applies a least squares fit directly to the finite element function and based on this fit a continuous gradient is determined. As we will show below, such a gradient recovery method results in a gradient approximation that satisfies all the conditions imposed on a quasi-normal field $\left(\right.$ on $\Gamma_{h}$ ) formulated in Definition 3.1. Such a gradient recovery technique is the first key component in our method. The second one is the approximate signed distance function introduced in Definition 3.2, which depends on the quasi-normal field resulting from the gradient recovery technique.

In section 6.1 we describe one paricular gradient recovery method known from the literature, namely the PPR method. We emphasize, however, that in the initialization method one could replace the PPR method by another gradient recovery algorithm. In section 6.2 we introduce our new initialization phase. In section 7 we apply the general results derived for the approximate signed distance function in section 4 to the finite element setting.

6.1. The polynomial-preserving recovery (PPR) method. The reason that we use PPR is that for this method more theoretical analysis, e.g. error bounds as in [9], is known. As mentioned already above, in the initialization method one can replace the PPR method by another gradient recovery algorithm. The general description (i.e. $d=2,3, k \geq 1$ ) of the PPR method is given in [9]. To simplify the presentation, we restrict to the cases $d=3, k=1,2$. For $v \in N\left(\mathcal{T}_{h}\right)$ let $\psi_{v}$ be the corresponding nodal finite element basis function. Below we explain how, given the set of function values $\left\{\phi_{h}(v) \mid v \in N\left(\mathcal{T}_{h}\right)\right\}$ the gradient recovery vectors $\left\{\left(G_{h} \phi_{h}\right)(v) \mid v \in N\left(\mathcal{T}_{h}\right)\right\}$ are constructed. The induced continuous gradient recovery finite element vector function is given by the operator $G_{h}: V_{h} \rightarrow V_{h}^{3}$ :

$$
G_{h} \phi_{h}:=\sum_{v \in N\left(\mathcal{T}_{h}\right)}\left(G_{h} \phi_{h}\right)(v) \psi_{v} \in V_{h}^{3}
$$

The function $G_{h} \phi_{h}$ is a continuous finite element approximation of the gradient: $\left(G_{h} \phi_{h}\right)(x) \approx \nabla \phi(x)$. In the application of the PPR method in section 6.2 we use the gradient recovery only "close to $\Gamma_{h}$ ". More precisely, we only need

$$
\left(G_{h} \phi_{h}\right)_{\mid \Omega_{\Gamma_{h}}}=\sum_{v \in N\left(\mathcal{T}_{\Gamma_{h}}\right)}\left(G_{h} \phi_{h}\right)(v) \psi_{v}
$$

It remains to explain how the vectors $\left(G_{h} \phi_{h}\right)(v), v \in N\left(\mathcal{T}_{\Gamma_{h}}\right)$ are determined. We first consider $k=1$. In this case the set of finite element nodes and the set of vertices coincide: $V\left(\mathcal{T}_{\Gamma_{h}}\right)=N\left(\mathcal{T}_{\Gamma_{h}}\right)$. Take $v \in V\left(\mathcal{T}_{\Gamma_{h}}\right)$ and the corresponding neigborhood $\omega(v)$. Let $p_{v}$ be the polynomial of degree 2 that fits $\phi_{h}$ in a least-squares sense:

$$
\sum_{z \in V(\omega(v))} \mid\left(\phi_{h}(z)-\left.p_{v}(z)\right|^{2}=\min _{p \in \mathcal{P}_{2}} \sum_{z \in V(\omega(v))} \mid\left(\phi_{h}(z)-\left.p(z)\right|^{2}\right.\right.
$$


We assume that this least-squares problem has a unique solution and define

$$
\left(G_{h} \phi_{h}\right)(v):=\nabla p_{v}(v) .
$$

Note that the polynomial $p_{v}$ is uniquely defined if we have at least 10 independent conditions. In our applications this holds, since we typically have 11-14 edges that have $v$ as a vertex. If there are not enough independent conditions one can enlarge the neighborhood $\omega(v)$ by including further neighboring simplices. This approach is explained in [9]. For ease of implementation we propose to use a simpler (but theoretically less favorable) approach. In case of less than 10 independent conditions we fit a polynomial of degree 2 (or 1) with less degrees of freedom. For example, we set the coefficient corresponding to $x y$ and/or $x z$ and/or $y z$ to zero. Another possibility is to use a local refinement of one or more of the $T \in \omega(v)$ and thus create more conditions.

We now consider $k=2$, and thus $V\left(\mathcal{T}_{\Gamma_{h}}\right) \neq N\left(\mathcal{T}_{\Gamma_{h}}\right)$. We first take $v \in V\left(\mathcal{T}_{\Gamma_{h}}\right)$. The approach is the same as above: we fit a polynomial $p_{v}$ of degree 3 to the values in all nodes in $\omega(v)$ :

$$
\sum_{z \in N(\omega(v))} \mid\left(\phi_{h}(z)-\left.p_{v}(z)\right|^{2}=\min _{p \in \mathcal{P}_{3}} \sum_{z \in N(\omega(v))} \mid\left(\phi_{h}(z)-\left.p(z)\right|^{2} .\right.\right.
$$

We assume a unique solution (which is generally true in our applications) and define

$$
\left(G_{h} \phi_{h}\right)(v):=\nabla p_{v}(v)
$$

We now consider $v \in N\left(\mathcal{T}_{\Gamma_{h}}\right) \backslash V\left(\mathcal{T}_{\Gamma_{h}}\right)$. Let $v$ be the midpoint of an edge with vertices $v_{1}$ and $v_{2}$. We define

$$
\left(G_{h} \phi_{h}\right)(v):=\frac{1}{2}\left(\nabla p_{v_{1}}(v)+\nabla p_{v_{1}}(v)\right)
$$

where $p_{v_{i}}$ are the least-squares polynomials defined in (6.4).

6.2. The initialization phase. As input for the method we need (only) $\mathcal{T}_{\Gamma_{h}}^{e}$ and $\left\{\phi_{h}(v) \mid v \in N\left(\mathcal{T}_{\Gamma_{h}}^{e}\right)\right\}$. Note that we do not need an approximation of $\Gamma_{h}$. The output is a finite element function $I_{h} d_{h} \in V_{h}\left(\mathcal{T}_{\Gamma_{h}}\right)$ that is "close to" the (local) signed distance function to $\Gamma_{h}$.

Given $\left\{\phi_{h}(v) \mid v \in N\left(\mathcal{T}_{\Gamma_{h}}^{e}\right)\right\}$, the PPR method described in (6.3) results in $G_{h} \phi_{h} \in V_{h}\left(\mathcal{T}_{\Gamma_{h}}^{e}\right)^{3}$, with $\left(G_{h} \phi_{h}\right)(x) \approx \nabla \phi(x)$ for $x \in \Omega_{\Gamma_{h}}$ (cf. section 7$)$. We define

$$
n_{h}(x)=\left\|\left(G_{h} \phi_{h}\right)(x)\right\|^{-1}\left(G_{h} \phi_{h}\right)(x), \quad x \in \Omega_{\Gamma_{h}} .
$$

As we will indicate in section 7 , it is reasonable to assume that this function satisfies the conditions of a quasi-normal field on $\Gamma_{h}$ as formulated in Definiton 3.1. Hence, for $z$ close to $\Gamma_{h}$ the approximate signed distance function $d(z)$, cf. Definition 3.2, is well-defined. To emphasize the dependence on the triangulation $\mathcal{T}_{h}$, we write $d_{h}$ instead of $d$. For $h \downarrow 0$ the points $z \in \Omega_{\Gamma_{h}}$ can be forced to be sufficiently close to $\Gamma_{h}$. We assume that $h$ is small enough such that the approximate signed distance function $d_{h}(z)$ is well-defined for $z \in \Omega_{\Gamma_{h}}$. Note that the function $d_{h}$ is close to the exact signed distance function to $\Gamma_{h}$, cf. Theorems 4.2 and 4.3 , and it has $\Gamma_{h}$ as its zero level:

$$
\left\{x \in \Omega_{\Gamma_{h}} \mid d_{h}(x)=0\right\}=\Gamma_{h} .
$$


Clearly, $d_{h}(z), z \in \Omega_{\Gamma_{h}}$, is not explicitly available. Therefore we introduce a nodal interpolation $I_{h} d_{h} \in V_{h}\left(\mathcal{T}_{\Gamma_{h}}\right)$ of $d_{h}$ given by

$$
I_{h} d_{h}(v)=d_{h}(v) \text { for all } v \in N\left(\mathcal{T}_{\Gamma_{h}}\right) .
$$

This finite element function $I_{h} d_{h}$ is the output of our initialization phase. Clearly, the interpolation procedure introduces an interpolation error that causes a change in the zero level, i.e.

$$
\tilde{\Gamma}_{h}:=\left\{x \in \Omega_{\Gamma_{h}} \mid\left(I_{h} d_{h}\right)(x)=0\right\} \approx \Gamma_{h} .
$$

As we will show below, this interpolation error can be considered to be "small" cf. Theorem (7.7).

It remains to discuss how $d_{h}(v), v \in N\left(\mathcal{T}_{\Gamma_{h}}\right)$, can be computed. For this we use an obvious variant of the method explained in section 5. Given a node $v \in N\left(\mathcal{T}_{\Gamma_{h}}\right)$ and $x^{k} \in \Gamma_{h}, x^{k} \approx p(v)$, we have to determine the zero of

$$
\phi_{h}\left(v-\alpha n_{h}\left(x^{k}\right)\right)=0,
$$

cf. (5.1). This can be realized by, for example, the following approximate Newton line-search: $\alpha_{0}:=0$ and

$$
\alpha_{n+1}=\alpha_{n}+\frac{\phi_{h}\left(v-\alpha_{n} n_{h}\left(x^{k}\right)\right)}{\left(G_{h} \phi_{h}\right)\left(v-\alpha_{n} n_{h}\left(x^{k}\right) \cdot n_{h}\left(x^{k}\right)\right.} \quad, n=0,1, \ldots
$$

The iteration in (5.1) takes the form

$$
x^{k+1}=v-\alpha n_{h}\left(x^{k}\right),
$$

with $\alpha$ that solves (6.8). A starting vector $x^{0} \in \Gamma_{h}$ can be determined as follows. Notice that the quasi-normal field $n_{h}$ is defined not only on $\Gamma_{h}$ but also on $\Omega_{\Gamma_{h}}$. Hence, one can define $x^{0}:=v-\alpha n_{h}(v)$, with $\alpha$ such that $\phi_{h}\left(v-\alpha n_{h}(v)\right)=0$ holds.

In the iterations (6.9), (6.10) the work per iteration is "low" since one only has to evaluate the known FE functions $\phi_{h}, G_{h} \phi_{h}$ and $n_{h}$. These iterations converge if $v$ is sufficiently close to $\Gamma_{h}$, cf. section 5 . Furthermore, the contraction of the iteration (6.10) can be bounded by $c|d(v)|$, cf. Theorem 5.1 for the precise statement. In the initialization phase we have $v \in N\left(\mathcal{T}_{\Gamma_{h}}\right)$ and thus $|d(v)|=\mathcal{O}(h)$, i.e. we have "fast" convergence for small $h$.

The iteration (6.10) is stopped when a suitable tolerance criterion is satisfied. The oublique projection of $v$ is then given by $p(v)=x^{k+1}$.

Concerning the practical realization of the algorithm there are several issues that should be addressed. For example. it might be better to replace (6.9) by a damped variant to guarantee that the iterates remain in $\Omega_{\Gamma_{h}}$. Furthermore it might be necessary to choose a better starting value than $x^{0}:=v-\alpha n_{h}(v)$ proposed above. These and other numerical issues will be investigated in a forthcoming paper.

7. Analysis of the PPR based initialization phase. In this section we use the results derived in section 4 to analyze the initialization phase presented in section 6.2. We consider the following setting. We assume that $\Gamma$ is the zero level of a level set function $\phi$, i.e. $\Gamma=\{x \in \Omega \mid \phi(x)=0\}$. The analysis is restricted to a (small) neighborhood $U \subset \Omega$ of $\Gamma$. We assume that $\phi$ is smooth on $U\left(\phi \in C^{k+1}(U)\right)$ and that there are constants $c_{L}>0$ and $c_{U}$ such that

$$
c_{L} \leq\|\nabla \phi(x)\| \leq c_{U} \quad \text { for all } x \in U .
$$


Let there be given a finite element function $\phi_{h} \in V_{h}$ that is an optimal approximation of $\phi$ in the neighborhood $U$, in the sense that

$$
\left\|\phi-\phi_{h}\right\|_{L^{\infty}(U)}+h\left\|\phi-\phi_{h}\right\|_{W^{1, \infty}(U)} \lesssim h^{k+1}
$$

holds. Here and in the remainder we use the notation $a \lesssim b$ to denote $a \leq c b$ with a constant $c$ independent of $h$. In applications, the finite element function $\phi_{h}$ is known and used as input for the initialization phase. The zero level of $\phi_{h}$, which for $k \geq 2$ can not be determined explicitly, is denoted by

$$
\Gamma_{h}=\left\{x \in \Omega \mid \phi_{h}(x)=0\right\} .
$$

Under mild assumptions, cf. section 7.2, we have

$$
\operatorname{dist}\left(\Gamma_{h}, \Gamma\right) \lesssim h^{k+1} .
$$

As output of the initialization phase we obtain $I_{h} d_{h} \in V_{h}$ as in (6.6), with a zero level denoted by

$$
\tilde{\Gamma}_{h}=\left\{x \in \Omega \mid\left(I_{h} d_{h}\right)(x)=0\right\} .
$$

Note that $\tilde{\Gamma}_{h}$ can also not be determined explicitly for $k \geq 2$.

In section 7.1 we show that the scaled recovered gradient (6.5) forms a quasinormal field on $\Gamma_{h}$ (but not on $\Gamma$ !) and that for the orthogonality measure $\delta_{x}$ in (3.3) we have $\delta_{x} \lesssim h^{k}$. Using this and the analysis in section 4 we derive an estimate that quantifies in which sense the output $I_{h} d_{h}$ of the initialization phase is "close to" the exact signed distance function to $\Gamma_{h}$, cf. Theorem 7.3. Furthermore, we show that $\tilde{\Gamma}_{h}$ has optimal accuracy, namely $\operatorname{dist}\left(\tilde{\Gamma}_{h}, \Gamma\right) \lesssim h^{k+1}$. The precise result is given in Theorem 7.7.

7.1. Recovered gradient as a quasi-normal field on $\Gamma_{h}$. In this section we assume that (7.1) and (7.2) hold. In the analysis we also need certain properties of the PPR gradient recovery operator $G_{h}: V_{h} \rightarrow V_{h}^{d}$. This operator has been analyzed in $[20,9]$. In our analysis we need the following two approximation and stability properties:

$$
\begin{aligned}
\left\|G_{h}\left(I_{h} \phi\right)-\nabla \phi\right\|_{L^{\infty}(U)} & \lesssim h^{k+1} \\
\left\|G_{h} v_{h}\right\|_{L^{\infty}(U)} & \leq c\left\|v_{h}\right\|_{W^{1, \infty}\left(U^{e}\right)} \quad \text { for all } \quad v_{h} \in V_{h},
\end{aligned}
$$

with a constant $c$ independent of $v_{h}$ and $h$. Here $U^{e}$ denotes the neighborhood $U$ extended with a suitable patch of surrounding elements (we refer to [9] for the details). The approximation result (7.6) follows from the Bramble-Hilbert lemma and the fact that $G_{h}$ has the consistency property $G_{h}\left(I_{h} p\right)=\nabla p$ for all $p \in \mathcal{P}_{k}$. The stability property (7.7) is proved for $d=2$ (under mild assumptions on the triangulation $\mathcal{T}_{h}$ ) in [9]. A proof of this property for $d=3$ is not known to us.

In the remainder we assume that (7.6) and (7.7) hold. In the following two lemmas, we show that the scaled recovered gradient $n_{h}$ defined in (6.5) satisfies the two conditions (3.2) and (3.3) for a quasi-normal field on $\Gamma_{h}$.

Lemma 7.1. Consider $n_{h}$ as in (6.5). There exist constants $c$ and $h_{0}>0$ such that for all $h \leq h_{0}$ the following holds:

$$
\left\|n_{h}(x)-n_{h}(y)\right\| \leq c\|x-y\| \quad \text { for all } x \in \Gamma_{h}, \quad y \in B\left(x ; r_{x}\right),
$$


with $r_{x}$ sufficiently small such that $B\left(x ; r_{x}\right) \subset U$.

Proof. Take $x \in \Gamma_{h}$ and $y \in B\left(x ; r_{x}\right) \subset U$. Inserting the definition we get

$$
\begin{aligned}
\left\|n_{h}(x)-n_{h}(y)\right\| & =\left\|\frac{\left(G_{h} \phi_{h}\right)(x)}{\left\|\left(G_{h} \phi_{h}\right)(x)\right\|}-\frac{\left(G_{h} \phi_{h}\right)(y)}{\left\|\left(G_{h} \phi_{h}\right)(y)\right\|}\right\| \\
& \leq 2 \frac{\left\|\left(G_{h} \phi_{h}\right)(x)-\left(G_{h} \phi_{h}\right)(y)\right\|}{\left\|\left(G_{h} \phi_{h}\right)(x)\right\|} .
\end{aligned}
$$

For the term in the denominator we have

$$
G_{h} \phi_{h}=G_{h}\left(\phi_{h}-I_{h} \phi\right)+\left(G_{h}\left(I_{h} \phi\right)-\nabla \phi\right)+\nabla \phi,
$$

and using (7.6), (7.7), (7.1), (7.2) and the interpolation bound $\left\|\phi-I_{h} \phi\right\|_{W^{1, \infty}\left(U^{e}\right)} \lesssim h^{k}$ we get

$$
\begin{aligned}
\left\|\left(G_{h} \phi_{h}\right)(x)\right\| & \geq\|\nabla \phi(x)\|-c\left\|\phi_{h}-I_{h} \phi\right\|_{W^{1, \infty}\left(U^{e}\right)}-c\left\|G_{h}\left(I_{h} \phi\right)-\nabla \phi\right\|_{L^{\infty}(U)} \\
& \geq c_{L}-c h^{k} .
\end{aligned}
$$

Hence, for $h$ sufficiently small we have

$$
\left\|\left(G_{h} \phi_{h}\right)(x)\right\| \geq \frac{1}{2} c_{L} .
$$

The vector function $G_{h} v_{h} \in V_{h}^{3}$ is Lipschitz continuous and

$$
\left\|\left(G_{h} \phi_{h}\right)(x)-\left(G_{h} \phi_{h}\right)(y)\right\| \leq \int_{0}^{1}\left\|\nabla\left(G_{h} \phi_{h}\right)(x+t(x-y))\right\| d t\|x-y\|
$$

holds. We write $z:=x+t(x-y) \in B\left(x ; r_{x}\right)$ and note that

$$
\left\|\nabla\left(G_{h} \phi_{h}\right)(z)\right\| \leq\left\|\nabla\left(G_{h} \phi_{h}-I_{h}(\nabla \phi)\right)\right\|_{L^{\infty}(U)}+\left\|\nabla I_{h}(\nabla \phi)\right\|_{L^{\infty}(U)} .
$$

Using an inverse inequality and the boundedness of $I_{h}$ on $W^{1, \infty}(U)$ we get

$$
\left\|\nabla\left(G_{h} \phi_{h}\right)(z)\right\| \lesssim h^{-1}\left\|G_{h} \phi_{h}-I_{h}(\nabla \phi)\right\|_{L^{\infty}(U)}+1
$$

and using (7.6), (7.7) results in

$$
\begin{aligned}
\left\|\nabla\left(G_{h} \phi_{h}\right)(z)\right\| & \lesssim h^{-1}\left\|G_{h}\left(\phi_{h}-I_{h} \phi\right)\right\|_{L^{\infty}(U)}+h^{-1}\left\|G_{h}\left(I_{h} \phi\right)-\nabla \phi\right\|_{L^{\infty}(U)} \\
& +h^{-1}\left\|\nabla \phi-I_{h}(\nabla \phi)\right\|_{L^{\infty}(U)}+1 \\
& \lesssim h^{-1}\left\|\phi_{h}-I_{h} \phi\right\|_{W^{1, \infty}\left(U^{e}\right)}+h^{k}+1 \lesssim h^{k-1}+1 \lesssim 1 .
\end{aligned}
$$

Using this result in (7.11), in combination with (7.10) and (7.9) completes the proof.

Lemma 7.2. There exist constants $c_{1}, c_{2}$ and $h_{0}>0$ such that for $h \leq h_{0}$ and all $x \in \Gamma_{h}, y \in \Gamma_{h} \cap B\left(x ; r_{x}\right)$, with $r_{x}$ sufficiently small such that $B\left(x ; r_{x}\right) \subset U$, the following holds:

$$
\left|\left\langle n_{h}(x), x-y\right\rangle\right| \leq c_{1} h^{k}\|x-y\|+c_{2}\|x-y\|^{2} .
$$


Proof. Using the definition of $n_{h}$ and the lower bound in (7.10) results in

$$
\left|\left\langle n_{h}(x), x-y\right\rangle\right| \leq \frac{2}{c_{L}}\left|\left\langle\left(G_{h} \phi_{h}\right)(x), x-y\right\rangle\right| .
$$

Since $x \in \Gamma_{h}$ and $y \in \Gamma_{h}$ we have

$$
0=\phi_{h}(x)-\phi_{h}(y)=\int_{0}^{1}\left\langle\nabla \phi_{h}(x+t(y-x), x-y\rangle d t .\right.
$$

Using this relation we obtain

$$
\begin{aligned}
& \left\langle\left(G_{h} \phi_{h}\right)(x), x-y\right\rangle=\int_{0}^{1}\left\langle\left(G_{h} \phi_{h}\right)(x)-\left(G_{h} \phi_{h}\right)(x+t(y-x)), x-y\right\rangle d t \\
& +\int_{0}^{1}\left\langle\left(G_{h} \phi_{h}\right)(x+t(y-x))-\nabla \phi_{h}(x+t(y-x)), x-y\right\rangle d t
\end{aligned}
$$

From the Lipschitz continuity estimate (7.11)-(7.12), with $y$ replaced by $x+t(y-x) \in$ $B\left(x, r_{x}\right)$ we get

$$
\left|\left\langle\left(G_{h} \phi_{h}\right)(x)-\left(G_{h} \phi_{h}\right)(x+t(y-x)), x-y\right\rangle\right| \leq c\|x-y\|^{2} .
$$

For the second term on the right-hand side in (7.14) we get

$$
\begin{aligned}
& \left|\left\langle\left(G_{h} \phi_{h}\right)(x+t(y-x))-\nabla \phi_{h}(x+t(y-x)), x-y\right\rangle\right| \leq\left\|G_{h} \phi_{h}-\nabla \phi_{h}\right\|_{L^{\infty}(U)}\|x-y\| \\
& \leq\left(\left\|G_{h}\left(\phi_{h}-I_{h} \phi\right)\right\|_{L^{\infty}(U)}+\left\|G_{h}\left(I_{h} \phi\right)-\nabla \phi\right\|_{L^{\infty}(U)}+\left\|\nabla \phi-\nabla \phi_{h}\right\|_{L^{\infty}(U)}\right)\|x-y\| \\
& \lesssim\left(\left\|\phi_{h}-I_{h} \phi\right\|_{W^{1, \infty}\left(U^{e}\right)}+h^{k+1}+h^{k}\right)\|x-y\| \lesssim h^{k}\|x-y\| .
\end{aligned}
$$

Using this and (7.15) in (7.14) in combination with (7.13) completes the proof.

From the lemmas above we conclude that indeed $n_{h}$ defines a quasi-normal field on $\Gamma_{h}$ and that for the parameter $\delta_{x}$ in (3.3) we have $\delta_{x} \leq c h^{k}$ with a constant $c$ independent of $x \in \Gamma_{h}$.

7.2. Accuracy of the initialization phase. In the previous section we have shown that, under reasonable assumptions, the scaled recovered gradient $n_{h}$ defines a quasi-normal field on $\Gamma_{h}$ and that for $\delta_{x}$ as in (3.3) we have $\delta_{x} \leq c h^{k}$, with a constant $c$ independent of $h$ and $x$. In this section, we use this result for $n_{h}$ and apply the results derived in section 4 to derive properties of the signed distance function $d_{h}$ and the zero level $\tilde{\Gamma}_{h}$ given in (6.7).

The results in section 4, e.g. in the theorems 4.2 and 4.3, hold on a neighborhood $F(D)$ of the manifold. Using the fact that $\lim _{h \rightarrow 0} \operatorname{dist}\left(\Gamma_{h}, \Gamma\right)=0$ (follows from (7.2)) and the results in the lemmas 7.1 and 7.1 one can check that the width of this neigborhood $F(D)$ around $\Gamma_{h}$ is bounded from below by a strictly positive constant uniformly for $h \rightarrow 0$. Hence, for $h$ sufficiently small the local neighborhood $\Omega_{\Gamma_{h}}$, that has width $\mathcal{O}(h)$ is contained in the neighborhood $F(D)$.

Let $d_{h}^{e x}$ denote the exact signed distance function to $\Gamma_{h}$. As output of the initialization phase we obtain $I_{h} d_{h}$, cf. (6.6). In the next theorem, which is an immediate consequence of Theorem 4.3, we quantify the statement, that $I_{h} d_{h}$ is "close to" the exact signed distance function to $d_{h}^{e x}$. 
THEOREM 7.3. There exist constants $c$ and $h_{0}>0$ such that for $h \leq h_{0}$ the following holds:

$$
0 \leq \frac{\left|I_{h} d_{h}(v)\right|-\left|d_{h}^{e x}(v)\right|}{\left|d_{h}^{e x}(v)\right|} \leq c h^{k}
$$

for all nodes $v \in N\left(\mathcal{T}_{\Gamma_{h}}\right), v \notin \Gamma_{h}$, that fulfill the condition, specified in Theorem 4.3, that $L_{x_{0}, v} \cap N_{s}$ has $1 D$ measure zero.

Proof. We apply Theorem 4.3. For $h$ sufficiently small we have $v \in F(D)$. For $v \in N\left(\mathcal{T}_{\Gamma_{h}}\right)$ we have $I_{h} d_{h}(v)=d_{h}(v)$. Due to Lemma 7.2 the parameter $\delta_{L}$ in Theorem 4.3 is bounded by $c h^{k}$.

In the generic case the condition " $L_{x_{0}, v} \cap N_{s}$ has $1 \mathrm{D}$ measure zero" used in the theorem above is satisfied. In the remainder of this section we analyze the accuracy of the perturbed zero level $\tilde{\Gamma}_{h}$ compared to that of $\Gamma_{h}$. We start with the accuracy of the latter as an approximation of the zero level $\Gamma$ of the "exact" level set solution $\phi$. The exact signed distance function to $\Gamma$ is denoted by $d^{e x}$. The exact normal field on $\Gamma$ is denoted by $n_{\Gamma}(x), x \in \Gamma$. We take a (sufficiently small) neighborhood $U$ of $\Gamma$ such that for all $z \in U$ there exists a unique $p(z)=x \in \Gamma$ such that $z=p(z)+d^{e x}(z) n_{\Gamma}(p(z))$, cf. (3.10). Furthermore, to simplify the analysis, we assume that $\Gamma_{h} \subset U$ is the graph of a function on $\Gamma$ in the following sense: there exists a function $g: \Gamma \rightarrow \mathbb{R}$ such that $\Gamma_{h}=\left\{x+g(x) n_{\Gamma}(x) \mid x \in \Gamma\right\}$. Then $d^{e x}(z)=g(p(z))$ holds for all $z \in \Gamma_{h}$.

Lemma 7.4. Define $c_{H}:=\max _{z \in \bar{U}}\left\|\nabla^{2} \phi(z)\right\|<\infty$ Assume that (7.1) and (7.2) are satisfied and that $\left|d^{e x}(z)\right| \leq c_{L} c_{H}^{-1}$ holds for all $z \in \Gamma_{h}$. For $h_{0}>0$ sufficiently small and $h \leq h_{0}$ the following holds:

$$
\operatorname{dist}\left(\Gamma_{h}, \Gamma\right):=\max _{z \in \Gamma_{h}}\left|d^{e x}(z)\right| \lesssim h^{k+1} .
$$

Proof. Take $z=p(z)+d^{e x}(z) n_{\Gamma}(p(z)) \in \Gamma_{h}$. Hence, $\left|d^{e x}(z)\right|=\|z-p(z)\|$ holds. From Taylor expansion we obtain

$$
\phi(z)-\phi(p(z))=\nabla \phi(p(z))^{T}(z-p(z))+\frac{1}{2}(z-p(z))^{T} \nabla^{2} \phi(\xi)(z-p(z)),
$$

for suitable $\xi$. Since $\nabla \phi(p(z))$ and $z-p(z)$ are aligned we obtain, using $\phi(p(z))=$ $\phi_{h}(z)=0$ :

$$
\begin{aligned}
\|\nabla \phi(p(z))\|\|z-p(z)\| & =\left|\nabla \phi(p(z))^{T}(z-p(z))\right| \\
& \leq\|\phi(z)-\phi(p(z))\|+\frac{1}{2} c_{H}\|z-p(z)\|^{2} \\
& =\left\|\phi(z)-\phi_{h}(z)\right\|+\frac{1}{2} c_{H}\|z-p(z)\|^{2} \\
& \leq c h^{k+1}+\frac{1}{2} c_{H}\|z-p(z)\|^{2} .
\end{aligned}
$$

From (7.1), (7.2) and $\|z-p(z)\| \leq c_{L} c_{H}^{-1}$ we then get $\|z-p(z)\| \lesssim h^{k+1}$.

In the next lemma we show that from the result in (7.17) it follows that the signed distance functions $d_{h}^{e x}$ and $d^{e x}$ to $\Gamma_{h}$ and $\Gamma$, respectively, are close.

Lemma 7.5. Let the assumptions as in Lemma 7.5 be fulfilled. For $h_{0}>0$ sufficiently small and $h \leq h_{0}$ the following holds:

$$
\left\|d_{h}^{e x}-d^{e x}\right\|_{L^{\infty}(U)} \lesssim h^{k+1} .
$$


Proof. Take $z \in U$. First we consider the case that $d_{h}^{e x}(z)$ and $d^{e x}(z)$ have the same sign. Then $\left|d_{h}^{e x}(z)-d^{e x}(z)\right|=|| d_{h}^{e x}(z)|-| d^{e x}(z)||$ holds. Let $z_{h}^{*} \in \Gamma_{h}$ be such that $\left|d_{h}^{e x}(z)\right|=\left\|z-z_{h}^{*}\right\|$. Since $p\left(z_{h}^{*}\right) \in \Gamma$, we get, using (7.17):

$$
\left|d^{e x}(z)\right|-\left|d_{h}^{e x}(z)\right| \leq\left\|z-p\left(z_{h}^{*}\right)\right\|-\left\|z-z_{h}^{*}\right\| \leq\left\|z_{h}^{*}-p\left(z_{h}^{*}\right)\right\|=\left|d^{e x}\left(z_{h}^{*}\right)\right| \lesssim h^{k+1} .
$$

Take $y_{h}^{*}:=p(z)+g(p(z)) n_{\Gamma}(p(z)) \in \Gamma_{h}$. With (7.17) we obtain

$$
\begin{aligned}
\left|d_{h}^{e x}(z)\right|-\left|d^{e x}(z)\right| & \leq\left\|z-y_{h}^{*}\right\|-\|z-p(z)\| \\
& \leq|g(p(z))|=\left|g\left(p\left(y_{h}^{*}\right)\right)\right|=\left|d^{e x}\left(y_{h}^{*}\right)\right| \lesssim h^{k+1} .
\end{aligned}
$$

Now we treat the case that $d_{h}^{e x}(z)$ and $d^{e x}(z)$ have opposite sign. With $y_{h}^{*}$ as defined above we get

$$
\left|d_{h}^{e x}(z)-d^{e x}(z)\right|=\|z-p(z)\|+\min _{x \in \Gamma_{h}}\|z-x\| \leq\|z-p(z)\|+\left\|z-y_{h}^{*}\right\| .
$$

Due to the sign property, $z$ is located on the line segment that connects $p(z)$ with $y_{h}^{*}$. Hence,

$$
\left|d_{h}^{e x}(z)-d^{e x}(z)\right| \leq\left\|p(z)-y_{h}^{*}\right\|=|g(p(z))|=\left|g\left(p\left(y_{h}^{*}\right)\right)\right|=\left|d^{e x}\left(y_{h}^{*}\right)\right| \lesssim h^{k+1}
$$

holds.

We are now in a position to derive a bound for the difference between the approximate signed distance function $I_{h} d_{h} \in V_{h}$, that results from the initialization phase, and the signed distance functions $d^{e x}$ and $d_{h}^{e x}$ to the zero levels $\Gamma$ and $\Gamma_{h}$, respectively.

LEMma 7.6. Let the assumptions as in Lemma 7.5 be satisfied. In addition we assume (the generic case) that all $v \in N\left(\mathcal{T}_{\Gamma_{h}}\right)$ fulfill the condition that $L_{x_{0}, v} \cap N_{s}$ has $1 D$ measure zero (cf. Theorem 4.3). For $h_{0}>0$ sufficiently small and $h \leq h_{0}$ the following holds:

$$
\begin{aligned}
&\left\|I_{h} d_{h}-d^{e x}\right\|_{L^{\infty}\left(\Omega_{\Gamma_{h}}\right)} \lesssim h^{k+1} \\
&\left\|I_{h} d_{h}-d_{h}^{e x}\right\|_{L^{\infty}\left(\Omega_{\Gamma_{h}}\right)} \lesssim h^{k+1} .
\end{aligned}
$$

Proof. From Theorem 4.3 it follows that ||$I_{h} d_{h}(v)|-| d_{h}^{e x}(v)|| \lesssim h^{k+1}$ holds, uniformly for $v \in N\left(\mathcal{T}_{\Gamma_{h}}\right)$. Since per construction $I_{h} d_{h}(v)$ and $d_{h}^{e x}(v)$ have the same sign, we get

$$
\max _{v \in N\left(\mathcal{T}_{\Gamma_{h}}\right)}\left|d_{h}(v)-d_{h}^{e x}(v)\right| \lesssim h^{k+1}
$$

and thus,

$$
\left\|I_{h}\left(d_{h}-d_{h}^{e x}\right)\right\|_{L^{\infty}\left(\Omega_{\Gamma_{h}}\right)} \leq c \max _{v \in N\left(\mathcal{T}_{\Gamma_{h}}\right)}\left|d_{h}(v)-d_{h}^{e x}(v)\right| \lesssim h^{k+1} .
$$

From the smoothness of $d^{e x}$ (close to $\Gamma$ ) we get $\left\|I_{h} d^{e x}-d^{e x}\right\|_{L^{\infty}\left(\Omega_{\Gamma_{h}}\right)} \lesssim h^{k+1}$. Combining these results with the result in (7.18) we get:

$$
\begin{aligned}
\left\|I_{h} d_{h}-d^{e x}\right\|_{L^{\infty}\left(\Omega_{\Gamma_{h}}\right)} & \leq\left\|I_{h}\left(d_{h}-d_{h}^{e x}\right)\right\|_{L^{\infty}\left(\Omega_{\Gamma_{h}}\right)}+\left\|I_{h}\left(d_{h}^{e x}-d^{e x}\right)\right\|_{L^{\infty}\left(\Omega_{\Gamma_{h}}\right)} \\
& +\left\|I_{h} d^{e x}-d^{e x}\right\|_{L^{\infty}\left(\Omega_{\Gamma_{h}}\right)} \lesssim h^{k+1}+\left\|d_{h}^{e x}-d^{e x}\right\|_{L^{\infty}\left(\Omega_{\Gamma_{h}}\right)} \lesssim h^{k+1},
\end{aligned}
$$


which completes the proof of (7.19). The result in (7.20) follows from (7.18) and (7.19).

As a direct consequence of Lemma 7.6 we obtain a result on the accuracy of the zero level $\tilde{\Gamma}_{h}$ of $I_{h} d_{h}$, cf. $(7.5)$.

THEOREM 7.7. Let the assumptions of Lemma 7.6 be fulfilled. For $h_{0}>0$ sufficiently small and $h \leq h_{0}$ the following holds:

$$
\begin{aligned}
\operatorname{dist}\left(\tilde{\Gamma}_{h}, \Gamma\right) & :=\max _{z \in \tilde{\Gamma}_{h}}\left|d^{e x}(z)\right| \lesssim h^{k+1} \\
\operatorname{dist}\left(\tilde{\Gamma}_{h}, \Gamma_{h}\right) & :=\max _{z \in \tilde{\Gamma}_{h}}\left|d_{h}^{e x}(z)\right| \lesssim h^{k+1} .
\end{aligned}
$$

Proof. Take $z \in \tilde{\Gamma}_{h}$, hence $\left(I_{h} d_{h}\right)(z)=0$. Using (7.19) we obtain,

$$
\left|d^{e x}(z)\right|=\left|\left(I_{h} d_{h}\right)(z)-d^{e x}(z)\right| \leq\left\|I_{h} d_{h}-d^{e x}\right\|_{L^{\infty}\left(\Omega_{\Gamma_{h}}\right)} \lesssim h^{k+1},
$$

which proves the result in (7.21). Using (7.20) the same argument can be applied to prove (7.22).

We conclude that under reasonable assumptions the PPR based initialization phase results in a reinitialization $I_{h} d_{h} \in V_{h}$, which locally (i.e. on $\Omega_{\Gamma_{h}}$ ) differs at most $\mathcal{O}\left(h^{k+1}\right)$ from the exact signed distance function $d_{h}^{e x}$ to $\Gamma_{h}$. Furthermore, the perturbation of the zero level caused by the reinitialization is also at most $\mathcal{O}\left(h^{k+1}\right)$.

8. Numerical experiment. In this section we present results of a numerical experiment. Related to the PPR based initialization algorithm introduced in section 6.2 there are several numerical issues that have to be studied. For example, the computational work needed in the PPR method and in the iterations (6.9)-(6.10), the robustness of these iterations w.r.t. the choice of the starting value and the rate of convergence of these iterations. These and other numerical aspects and the application of the algorithm to different test problems will be studied in a forthcoming paper. Here we consider only one test problem and illustrate the theoretical accuracy bounds derived in section 7.2.

For the manifold $\Gamma$ we take the torus, characterized by the distance function

$$
d^{e x}(x)=\sqrt{x_{3}^{2}+\left(\sqrt{x_{1}^{2}+x_{2}^{2}}-R\right)^{2}}-r,
$$

with radii $r=0.2$ and $R=0.4$. For computations $\Gamma$ is embedded in the domain $[-1,1]^{3}$. We construct finite element spaces as follows. First a uniform tetrahedral triangulation of $\Omega$ with mesh size parameter $h_{0}=2 / 3$ is constructed. This uniform grid is locally refined in a neigborhood of $\Gamma$ by using a red-green procedure. This results in triangulations with mesh size parameters $h_{\ell}=2^{-\ell} h_{0}, \ell=1,2, \ldots 8$, close to $\Gamma$. These triangulations are denoted by $\mathcal{T}_{\ell}$. A cross-section of the level $\ell=5$ triangulation is given in Fig. 8.1.

We use standard finite element spaces with polynomials of degree $k=1$ and $k=2$ :

$$
V_{\ell}^{k}=\left\{\psi \in C(\Omega) \mid \psi_{\mid T} \in \mathcal{P}_{k} \text { for all } T \in \mathcal{T}_{\ell}\right\}, \quad k=1,2 .
$$

The nodel interpolation corresponding to this space is denoted by $I_{h}$. As exact level set function we take a perturbation of $d^{e x}$, given by $\phi^{(\alpha)}=d^{e x} q^{(\alpha)}$, with

$$
q^{(\alpha)}(x)=9.0+4.0 \cos \left(\alpha x_{1} x_{2} /\|x\|_{2}\right), \quad \alpha \in\{1,10,50\} .
$$




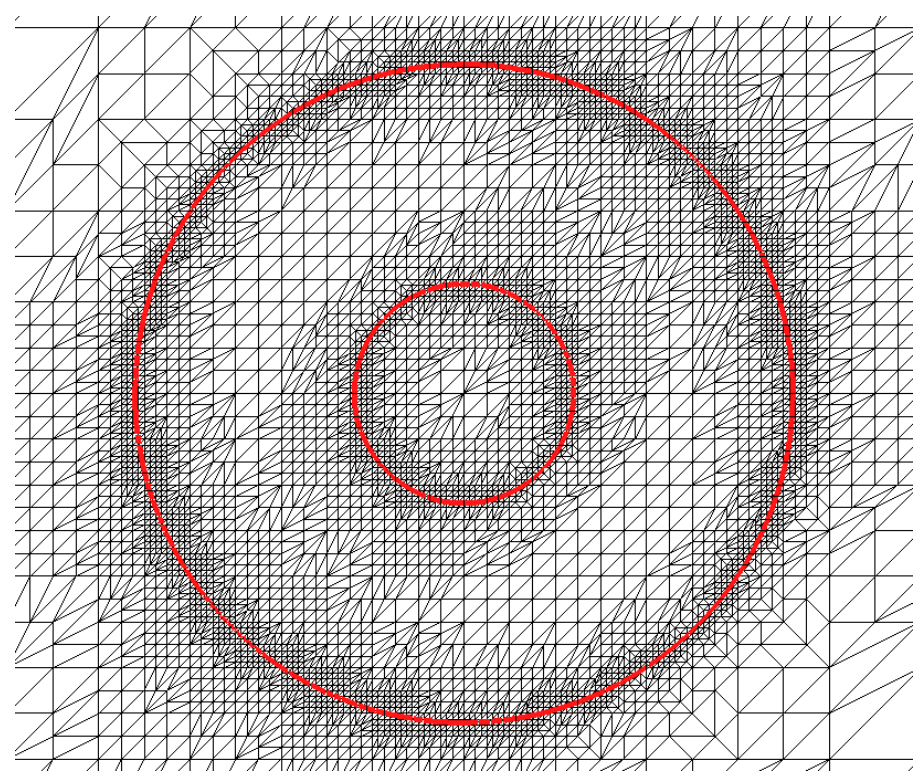

FIG. 8.1. Cross-section of tetrahedral triangulation on level $\ell=5$.

Note that $\phi^{(\alpha)}$ has $\Gamma$ as its zero level, it has a large gradient and for increasing $\alpha$ it becomes more and more distorted. For $\alpha=50$ the level lines of $\phi^{(\alpha)}$ on the same cross-section as in Fig. 8.1 are illustrated in Fig. 8.2. The four level lines shown in the figure correspond to the values $\phi^{(\alpha)}=-0.2,-0.1,0.1,0.2$.

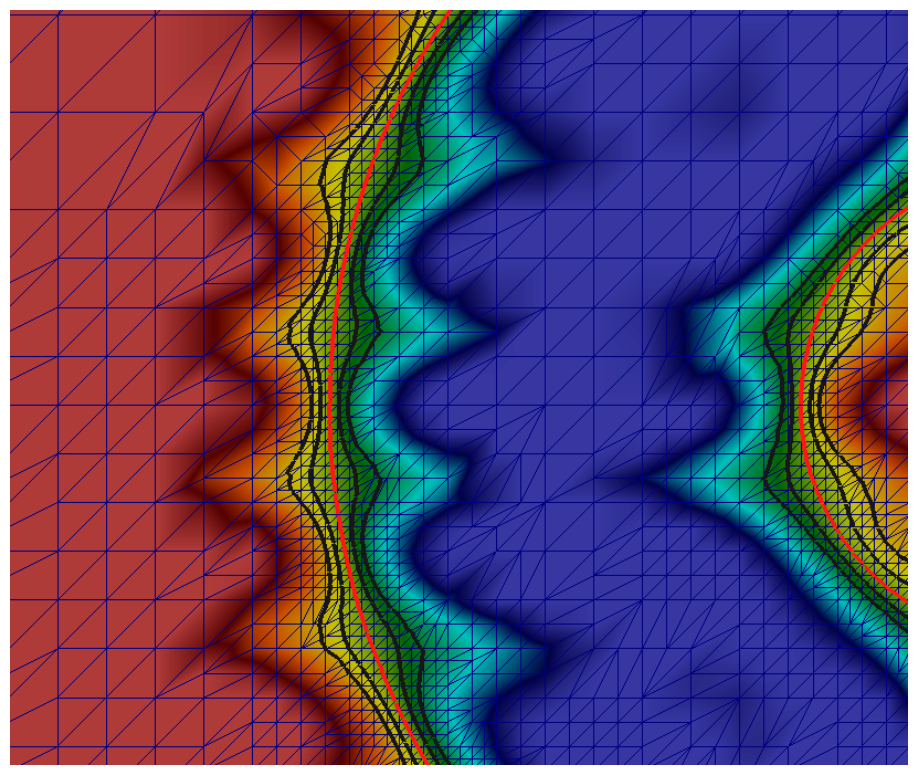

FIG. 8.2. Level lines of function $\phi^{(\alpha)}$ for $\alpha=50$.

As input for the initialization phase we take $\phi_{h}^{(\alpha)}=I_{h} \phi^{(\alpha)}$. Note that for $k=2$ the zero level $\Gamma_{h}$ of $\phi_{h}^{(\alpha)}$ can not be constructed explicitly. We implemented the 
initialization phase described in section 6.2. We summarize its main components:

- Based on sign properties the local triangulation $\mathcal{T}_{\Gamma_{h}}$ that contains $\Gamma_{h}$ is determined.

- For $v \in N\left(\mathcal{T}_{\Gamma_{h}}\right)$ the recovered gradient $\left(G_{h} \phi_{h}^{(\alpha)}\right)(v)$ and its normalization $n_{h}(v)=\left\|\left(G_{h} \phi_{h}^{(\alpha)}\right)(v)\right\|^{-1}\left(G_{h} \phi_{h}^{(\alpha)}\right)(v)$ are determined.

- For $v \in N\left(\mathcal{T}_{\Gamma_{h}}\right)$ the approximate signed distance value $d_{h}^{(\alpha)}(v)$ is determined by using (6.9)-(6.10).

- Nodal interpolation results in the finite element function $I_{h} d_{h}^{(\alpha)} \in V_{h}^{k}\left(\Omega_{\Gamma_{h}}\right)$, which is the output of the initialization phase.

Since the signed distance function $d_{h}^{e x}$ to $\Gamma_{h}$ (the zero level of $\phi_{h}^{(\alpha)}$ ) is not known, we compare the output $I_{h} d_{h}^{(\alpha)}$ to the signed distance function $d^{e x}$ to $\Gamma$. We use the following error measure:

$$
e_{\infty}:=\max _{v \in N\left(T_{\Gamma_{h}}\right)}\left|I_{h} d_{h}(v)-d^{\mathrm{ex}}(v)\right| .
$$

Furthermore, to measure the size of the gradient of $I_{h} d_{h}$ on $\Omega_{\Gamma_{h}}$ we determine

$$
e_{\infty}^{\nabla}:=\max _{T \in \mathcal{T}_{\Gamma_{h}}}\left|\sqrt{\frac{1}{|T|} \int_{T}\left\|\nabla\left(I_{h} d_{h}\right)(s)\right\|^{2} d s}-1\right| .
$$

The results are shown in the tables 8.1, 8.2, 8.3 below, for the cases $\alpha=1,10,50$, respectively.

\begin{tabular}{c|cc|ccc|cc|cc} 
& \multicolumn{2}{|c|}{$k=1$} & \multicolumn{2}{|c|}{$k=1$} & \multicolumn{2}{c|}{$k=2$} & \multicolumn{2}{c}{$k=2$} \\
$\ell$ & $e_{\infty}$ & order & $e_{\infty}^{\nabla}$ & order & $\ell$ & $e_{\infty}$ & order & $e_{\infty}^{\nabla}$ & order \\
\hline \hline 3 & $1.21 e-2$ & - & $2.50 e-1$ & - & 3 & $5.73 e-4$ & - & $1.66 e-2$ & - \\
4 & $3.01 e-3$ & 2.00 & $1.53 e-1$ & 0.71 & 4 & $7.21 e-5$ & 2.99 & $3.46 e-3$ & 2.26 \\
5 & $7.54 e-4$ & 2.00 & $8.32 e-2$ & 0.87 & 5 & $9.94 e-6$ & 2.86 & $8.70 e-4$ & 1.99 \\
6 & $1.90 e-4$ & 1.99 & $4.25 e-2$ & 0.97 & 6 & $1.24 e-6$ & 3.01 & $2.13 e-4$ & 2.03 \\
7 & $4.68 e-5$ & 2.02 & $2.12 e-2$ & 1.00 & 7 & $1.58 e-7$ & 2.96 & $5.31 e-5$ & 2.01 \\
8 & $1.19 e-5$ & 1.98 & $1.07 e-2$ & 0.99 & 8 & $2.07 e-8$ & 2.93 & $1.34 e-5$ & 1.98
\end{tabular}

Errors and order of convergence for $\alpha=1$.

\begin{tabular}{c|cc|ccc|cc|cc} 
& \multicolumn{2}{|c|}{$k=1$} & \multicolumn{2}{|c|}{$k=1$} & \multicolumn{2}{c|}{$k=2$} & \multicolumn{2}{c}{$k=2$} \\
$\ell$ & $e_{\infty}$ & order & $e_{\infty}^{\nabla}$ & order & $\ell$ & $e_{\infty}$ & order & $e_{\infty}^{\nabla}$ & order \\
\hline \hline 3 & $1.15 e-2$ & - & $2.40 e-1$ & - & 3 & $7.16 e-4$ & - & $1.77 e-2$ & - \\
4 & $3.24 e-3$ & 1.82 & $1.61 e-1$ & 0.57 & 4 & $1.04 e-4$ & 2.78 & $3.78 e-3$ & 2.23 \\
5 & $7.91 e-4$ & 2.03 & $8.02 e-2$ & 1.00 & 5 & $1.56 e-5$ & 2.75 & $1.10 e-3$ & 1.78 \\
6 & $2.07 e-4$ & 1.94 & $4.32 e-2$ & 0.89 & 6 & $1.87 e-6$ & 3.06 & $3.10 e-4$ & 1.83 \\
7 & $5.18 e-5$ & 2.00 & $2.24 e-2$ & 0.95 & 7 & $2.44 e-7$ & 2.94 & $7.32 e-5$ & 2.08 \\
8 & $1.32 e-5$ & 1.97 & $1.11 e-2$ & 1.01 & 8 & $3.09 e-8$ & 2.98 & $1.91 e-5$ & 1.94 \\
& \multicolumn{4}{|c}{ TABLE 8.2} & & &
\end{tabular}

Errors and order of convergence for $\alpha=10$.

In the cases indicated with $*$ in Table 8.3 there were convergence problems in the sense that the approximate Newton line-search (6.9) did not satisfy its tolerance criterion. 


\begin{tabular}{|c|c|c|c|c|c|c|c|c|c|}
\hline \multirow[b]{2}{*}{$\ell$} & \multicolumn{2}{|c|}{$k=1$} & \multicolumn{2}{|c|}{$k=1$} & \multirow[b]{2}{*}{$\ell$} & \multicolumn{2}{|c|}{$k=2$} & \multicolumn{2}{|c|}{$k=2$} \\
\hline & $e_{\infty}$ & order & $e_{\infty}^{\nabla}$ & order & & $e_{\infty}$ & order & $e_{\infty}^{\nabla}$ & order \\
\hline 3 & $4.07 e-2$ & - & $6.23 e-1$ & - & $3^{*}$ & $7.47 e-2$ & - & $8.10 e-1$ & - \\
\hline 4 & $1.05 e-2$ & 1.95 & $2.32 e-1$ & 1.42 & $4^{*}$ & $2.86 e-2$ & 1.38 & $3.10 e-1$ & 1.39 \\
\hline 5 & $2.91 e-3$ & 1.86 & $1.01 e-1$ & 1.21 & $5^{*}$ & $5.66 e-4$ & 5.66 & $1.63 e-2$ & 4.25 \\
\hline 6 & $7.63 e-4$ & 1.93 & $5.76 e-2$ & 0.80 & 6 & $6.87 e-5$ & 3.04 & $2.48 e-2$ & -0.61 \\
\hline 7 & $1.94 e-4$ & 1.98 & $3.04 e-2$ & 0.92 & 7 & $7.21 e-6$ & 3.25 & $1.52 e-3$ & 4.03 \\
\hline 8 & $4.84 e-5$ & 2.00 & $1.58 e-2$ & 0.94 & 8 & $8.78 e-7$ & 3.04 & $3.90 e-4$ & 1.97 \\
\hline
\end{tabular}

Errors and order of convergence for $\alpha=50$.

The results in the tables show an error reduction behavior that is consistent with the theoretical results derived in Lemma 7.6 for the error measure $e_{\infty}$ and with the results on the size of the gradient of the approximate signed distance function in Theorem 4.2. In the application of the latter theorem to our method we have $\delta_{p(z)} \leq c h^{k}$, cf. section 7.1.

Acknowledgement. The author acknowledges the help of P. Esser with the implementation of the method.

\section{REFERENCES}

[1] D. Adalsteinsson and J. Sethian, The fast construction of extension velocities in level set methods, J. Comp. Phys., 148 (1999), pp. 2-22.

[2] M. Ainsworth and J. Oden, A Posteriori Error Estimation in Finite Element Analysis, Wiley, New York, 2000.

[3] R. Ausus, E. Dari, and G. Buscaglia, A geometric mass-preserving redistancing scheme for the level set function, Int. J. Numer. Meth. Fluids, 65 (2011), pp. 989-010.

[4] D. Chopp, Computing minimal surfaces via level set curvature flow, J. Comp. Phys., 106 (1993), pp. 77-91.

[5] - Some improvements of the fast marching method, SIAM J. Sci.,Comput., 23 (2001), pp. 230-244.

[6] _ Another look at velocity extension in the level set method, SIAM J. Sci.,Comput., 31 (2009), pp. 3255-3273.

[7] F. Clarke, Optimization and Nonsmooth Analysis, Wiley, New York, 1983.

[8] P. Grisvard, Elliptic Problems in Nonsmooth Domains, Pitman Publishing Inc., Boston, 1985.

[9] A. NAGA AND Z. Zhang, The polynomial-preserving recovery for higher order finite element methhods in 2D and 3D, Discr. and Continuous Dynamical Systems - Series B, 5 (2005), pp. 769-798.

[10] J. Naumann And C. Simader, Measure and integration on Lipschitz-Manifolds, Preprint 07-15, Department of Mathematics, Humboldt University Berlin, 2007. http://edoc.huberlin.de/series/mathematik-preprints/2007-15/PDF/15.pdf.

[11] S. Osher AND R. P. Fedkiw, Level set methods: An overview and some recent results, J. Comp. Phys., 169 (2001), pp. 463-502.

[12] - Level Set Methods and Dynamic Implicit Surfaces, Springer, New York, 2003.

[13] J.-S. PANG AND L. QI, Nonsmooth equations: Motivation and algorithms, SIAM J. on Optimization, 3 (1993), pp. 443-465.

[14] G. Russo and P. Smereka, A remark on computing distance functions, J. Comp. Phys., 163 (2000), pp. 51-67.

[15] J. A. Sethian, A fast marching level set method for monotonically advancing fronts, in Proc. Natl. Acad. Sci. USA, vol. 93, 1996, pp. 1591-1595.

[16] - Theory, algorithms, and applications of level set methods for propagating interfaces, Acta Numerica, 5 (1996), pp. 309-395.

[17] —, Level Set Methods and Fast Marching Methods, Cambridge University Press, 1999.

[18] M. Sussman, A. S. Almgren, J. B. Bell, P. Colella, L. H. Howell, And M. L. Welcome, 
An adaptive level set approach for incompressible two-phase flows, J. Comp. Phys., 148 (1999), pp. 81-124.

[19] M. Sussman And E. Fatemi, An efficient, interface-preserving level set redistancing algorithm and its application to interfacial incompressible fluid flow, SIAM J. Sci. Comput., 20 (1999), pp. 1165-1191.

[20] Z. ZHANG AND A. NAGA, A new finite element gradient recovery method: superconvergence property, SIAM J. Sci. Comput., 26 (2005), pp. 1192-1213.

[21] O. ZiENKIEWICZ AND J. ZHU, The superconvergence patch recovery and a posteriori error estimates, part I: the recovery technique, Intern. J. Numer. Methods Engrg., 33 (1992), pp. 1331-1364.

[22] — The superconvergence patch recovery and a posteriori error estimates, part II: error estimates and adaptivity, Intern. J. Numer. Methods Engrg., 33 (1992), pp. 1365-1382. 